\title{
Attractor and Boundedness of Switched Stochastic Cohen-Grossberg Neural Networks
}

\author{
Chuangxia Huang, ${ }^{1,2}$ Jie Cao, ${ }^{1}$ and Peng Wang ${ }^{1}$ \\ ${ }^{1}$ School of Mathematics and Statistics, Changsha University of Science and Technology, Changsha, Hunan 410114, China \\ ${ }^{2}$ Academy of Mathematics and Systems Science, Chinese Academy of Sciences, Beijing 100190, China \\ Correspondence should be addressed to Chuangxia Huang; cxiahuang@126.com
}

Received 15 February 2016; Accepted 5 April 2016

Academic Editor: Guoqiang Hu

Copyright (c) 2016 Chuangxia Huang et al. This is an open access article distributed under the Creative Commons Attribution License, which permits unrestricted use, distribution, and reproduction in any medium, provided the original work is properly cited.

\begin{abstract}
We address the problem of stochastic attractor and boundedness of a class of switched Cohen-Grossberg neural networks (CGNN) with discrete and infinitely distributed delays. With the help of stochastic analysis technology, the Lyapunov-Krasovskii functional method, linear matrix inequalities technique (LMI), and the average dwell time approach (ADT), some novel sufficient conditions regarding the issues of mean-square uniformly ultimate boundedness, the existence of a stochastic attractor, and the mean-square exponential stability for the switched Cohen-Grossberg neural networks are established. Finally, illustrative examples and their simulations are provided to illustrate the effectiveness of the proposed results.
\end{abstract}

\section{Introduction}

In the last few decades, theoretical and applied researches of artificial neural networks have been the new worldwide focus. Some of the reasons for this are due to the successful hardware implementations and their various applications, such as classification, associative memories, parallel computation, optimization, and signal processing $[1,2]$. It is recognized that such applications of neural networks depend heavily on some dynamic behaviors, such as stability properties, periodic oscillatory behavior, and attractor and boundedness (see [3$16]$ and references therein).

Since the seminal work by Cohen and Grossberg [17], Cohen-Grossberg neural networks have been intensively studied [2, 18-22]. During hardware implementation, time delays do exist due to the finite switching speed of the amplifiers and communication time; it is important to incorporate delays into the neural networks. Generally speaking, there are two kinds of delays, discrete delays and distributed delays $[2,16,23]$. The utilization of discrete delays in models of delayed feedback provides a good approximation in simple circuits consisting of a small number of cells. When the neural networks have a spatial extent due to the presence of a multitude of parallel pathways with a variety of axon sizes and lengths, it is necessary to incorporate continuously distributed delays. The distributed delay includes finite delay and infinite delay $[2,5,18,24]$.

In real nervous systems, synaptic transmission is a noisy process brought about by random fluctuations from the release of neurotransmitters and other probabilistic causes $[19,25-27]$. It is well known that for stochastic neural networks, it is rather difficult to analyze their dynamic properties due to the introduction of noise. Such studies are however important for understanding the dynamic characteristics of neuron behavior in stochastic environments. For instance, during the implementation of Kalman filter training, stochastic neural networks characterized as zero-mean white noise have been successfully employed [2].

On the other hand, neural networks are complex and large-scale nonlinear dynamics; during hardware implementation, the connection topology of networks may change very quickly and link failures or new creations in networks often bring about switching connection topology [2]. 
To obtain a deep and clear understanding of the dynamics of this complex system, one of the usual ways is to investigate the switched neural network. As a special class of hybrid systems, switched neural network systems are composed of a family of continuous-time or discrete time subsystems and a rule that orchestrates the switching among the subsystems [28]. In general, the switched rule is a piecewise constant function dependent on the state or time. The logical rule that orchestrates switching between these subsystems generates switching signals [29]. Recently, switched systems have numerous applications in the control of mechanical systems, the automotive industry, aircraft and air traffic control, switching power converters, and many other fields [30]. In [28], Huang et al. are the first to investigate the robust stability of switched Hopfield neural networks with time-varying delays by an arbitrary switched rule. The average dwell time approach provided an effective tool to study the stability of switched systems. Wu et al. used average dwell time approach to analyze the exponential stability of continuoustime switched delayed neural networks in [31]. In [27], the average dwell time and LMI method have been utilized to discuss the exponential synchronization of switched stochastic competitive neural networks with mixed delays. In addition, [32] has focused on the delay-dependent global robust asymptotic stability problem of uncertain switched Hopfield neural networks (USHNNs) with discrete interval and distributed time-varying delays and time delay in the leakage term. Moreover, parametric uncertainty which often breaks the stability of systems can be commonly encountered due to modeling inaccuracies or changes in the environment of the model. To deal with the difficulties brought about by uncertainty, exponential stability analysis and $H_{\infty}$ control of different uncertain systems have received great research attention [33]. Moreover, the parametric uncertainty is assumed to be norm-bounded in [34]. Unfortunately, up to now, few researchers have considered the mean-square uniformly ultimate boundedness and stochastic attractor for switched SCGNN with discrete delays and infinite distributed delays.

However, these available literatures mainly consider the stability property of switching neural networks. In fact, except for the stability property, boundedness and attractor are also the foundational concepts of dynamical neural networks, which play important roles in the investigation of the uniqueness of the equilibrium point (periodic solutions), global asymptotic stability, global exponentially stability, and the synchronization [35]. To the best of the author's knowledge, few researchers have considered the uniformly ultimate boundedness and attractors for switched CGNN with discrete delays and distributed delays.

Inspired by the above discussions, the objects of this paper are to study the mean-square uniformly ultimate boundedness and stochastic attractor for switched SCGNN with discrete delays and infinitely distributed delays by employing stochastic analysis technology, the Lyapunov-Krasovskii functional method, the linear matrix inequalities (LMI) technique, and the average dwell time approach (ADT). In addition, the parametric uncertainty is considered and assumed to be norm-bounded.
As is well known, mean-square uniformly ultimate boundedness (MSUUB) conditions are derived in terms of linear matrix inequalities (LMIs), which can be easily calculated by the MATLAB LMI control toolbox. All of the above mentioned reasons motivate us to investigate the problems of the MSUUB and stochastic attractor for switched SCGNN in this paper. Numerical examples are provided to demonstrate the feasibility and effectiveness of the proposed criteria.

The rest of this paper is organized as follows. Some preliminaries are given in Section 2. We present some basic definitions and notations, as well as some lemmas needed in later sections. In Section 3, we present some sufficient conditions of MSUUB and stochastic attractor for switched stochastic CGNN. In Section 4, an example is presented to illustrate the effectiveness of the proposed approach. The conclusions are summarized in Section 5.

Notations. The superscript " $T$ " stands for matrix transposition; $R^{n}$ denotes the $n$-dimensional Euclidean space; the notation $P>0$ means that $P$ is real symmetric and positivedefinite; $I$ and $O$ represent the identity matrix and a zero matrix, respectively; $\operatorname{diag}\{\cdots\}$ stands for a block-diagonal matrix; and $\lambda_{\min }(P)\left(\lambda_{\max }(P)\right)$ denotes the minimum (maximum) eigenvalue of symmetric matrix $P$. In symmetric block matrices or long matrix expressions, a $(*)$ is used to represent a term that is induced by symmetry.

\section{Preliminaries and Problem Formulation}

The Itô's formula plays a key role in the dynamic analysis of stochastic systems. To facilitate understanding, some related results are cited here (see [36] for details). For a general stochastic system $d x(t)=f(x(t), t) d t+g(x(t), t) d w(t)$ on $t \geq t_{0}$ with initial value $x\left(t_{0}\right)=x_{0} \in R^{n}$, where $w(t)$ is $m$-dimensional Brownian motion defined on $(\Omega, \mathscr{F}, \mathscr{P})$, $f: R^{n} \times R^{+} \rightarrow R^{n}$, and $g: R^{n} \times R^{+} \rightarrow R^{n \times m}$. Let $\mathscr{C}^{1,2}\left(R^{n} \times\right.$ $R^{+} ; R^{+}$) be the family of all nonnegative functions which are continuous once differentiable in $t$ and twice differentiable in $x$. For $V \in \mathscr{C}^{1,2}\left(R^{n} \times R^{+} ; R^{+}\right)$, define an operator $\mathscr{L} V$ from $\left(R^{n} \times R^{+} ; R^{+}\right)$to $R$ by

$$
\begin{aligned}
d V & =\mathscr{L} V d t+\frac{\partial V}{\partial x} g(x(t), t) d \omega(t) \\
\mathscr{L} V(x(t), t) & \\
= & V_{t}(x(t), t)+V_{x}(x(t), t) f(x(t), t) \\
& +\frac{1}{2} \operatorname{tra}\left[g^{T}(x(t), t) V_{x x}(x(t), t) g(x(t), t)\right],
\end{aligned}
$$

and $V_{t}(x(t), t)=\partial V(x(t), t) / \partial t, V_{x x}(x(t), t)=\left(\partial^{2} V(x(t), t) /\right.$ $\left.\partial x_{i} \partial x_{j}\right)_{n \times n}$, and $V_{x}(x(t), t)=\left(\partial V(x(t), t) / \partial x_{1}, \ldots, \partial V(x(t)\right.$, t)/ $\left.\partial x_{n}\right)$.

In practical systems, the neural network models are disturbed by environmental noises. Therefore, in this paper, we will consider the stochastic Cohen-Grossberg neural 
networks with mixed time delay described by the following stochastic nonlinear integrodifferential equations:

$$
\begin{aligned}
& d x_{i}(t)=-\widehat{\alpha}_{i}\left(x_{i}(t)\right)\left[\widehat{\beta}_{i}\left(x_{i}(t)\right)-\sum_{j=1}^{n} a_{i j} f_{j}\left(x_{j}(t)\right)\right. \\
& -\sum_{j=1}^{n} b_{i j} f_{j}\left(x_{j}\left(t-\tau_{j}(t)\right)\right) \\
& \left.\quad-\sum_{j=1}^{n} c_{i j} \int_{-\infty}^{t} k_{i j}(t-s) f_{j}\left(x_{j}(s)\right) d s-J_{i}\right] d t \\
& +\sum_{j=1}^{n} \sigma_{i j}\left(t, x_{i}(t), x_{i}\left(t-\tau_{j}(t)\right)\right) d w_{j}(t), \\
& i=1, \ldots, n .
\end{aligned}
$$

System (2) for convenience can be rewritten as the following vector form:

$$
\begin{aligned}
& d x(t)=-\widehat{\alpha}(x(t))[\widehat{\beta}(x(t))-A F(x(t)) \\
& -B F(x(t-\tau(t))) \\
& \left.-C \int_{-\infty}^{t} K(t-s) F(x(s)) d s-J\right] d t \\
& +\sigma(t, x(t), x(t-\tau(t))) d w(t),
\end{aligned}
$$

where $A, B, C$ are known constant matrices with appropriate dimensions, $x(t)=\left[x_{1}(t), \ldots, x_{n}(t)\right]^{T} \in R^{n}$ is the neural state vector associated with the $n$ neurons at time $t, \widehat{\alpha}(x(t))=$ $\operatorname{diag}\left[\widehat{\alpha}_{1}\left(x_{1}(t)\right), \ldots, \widehat{\alpha}_{n}\left(x_{n}(t)\right)\right] \in R^{n \times n}$ represents an amplification function, $\widehat{\beta}(x(t))=\left[\widehat{\beta}_{1}\left(x_{1}(t)\right), \ldots, \widehat{\beta}_{n}\left(x_{n}(t)\right)\right] \in R^{n \times n}$ denotes the behavior function, $F(x(t))=\left[F_{1}\left(x_{1}(t)\right), \ldots\right.$, $\left.F_{n}\left(x_{n}(t)\right)\right]^{T}$ is the neuron activation functions, $K(t-s)=$ $\operatorname{diag}\left[k_{1}(t-s), k_{2}(t-s), \ldots, k_{n}(t-s)\right], \tau(t)=\left[\tau_{1}(t), \ldots, \tau_{n}(t)\right]^{T}$, $J=\left[J_{1}, \ldots, J_{n}\right]^{T}$ is the constant external input vector, $\sigma(t)=$ $\left(\sigma_{i j}(t)\right)_{n \times n}$ is the diffusion coefficient matrix, and $w(x(t))=$ $\left[w_{1}\left(x_{1}(t)\right), \ldots, w_{n}\left(x_{n}(t)\right)\right]^{T}$ is an $n$-dimension Brownian motion satisfying $E\{d \omega(t)\}=0, E\left\{d \omega^{2}(t)\right\}=d t$ and defined on a complete probability space $(\Omega, \mathscr{F}, \mathscr{P})$ with a natural filtration $\left\{\mathscr{F}_{t}\right\}_{t \geq 0}$ generated by $\{w(s): 0 \leq s \leq t\}$, where we associate $\Omega$ with the canonical space generated by $w(t)$ and denote by $\mathscr{F}$ the associated $\sigma$-algebra generated by $\{w(t)\}$ with the probability measure $\mathscr{P}$.

The discrete time-varying delays $\tau(t)$ satisfy

$$
\begin{aligned}
0 & \leq \tau_{m} \leq \tau(t) \leq \tau_{M}<+\infty, \\
\dot{\tau}(t) & <\mu,
\end{aligned}
$$

and the delay kernel $k_{j}$ is a real valued continuous function defined on $[0,+\infty]$ and satisfies; for each $j$,

$$
\int_{0}^{\infty} k_{j}(s) d s=1 .
$$

Moreover, there exist $\iota>0$ and matrix $\underline{K}=\operatorname{diag}\left\{\underline{k}_{1}(\iota)\right.$, $\left.\underline{k}_{2}(l), \ldots, \underline{k}_{n}(l)\right\}>0$,

$$
\int_{0}^{\infty} k_{j}(s) e^{\iota s} d s=\underline{k}_{j}(\iota) .
$$

As usual, the initial conditions associated with system (3) are given in the form

$$
x(t)=\varphi(t), \quad-\infty<t \leq 0,
$$

where the initial value function $\varphi \in L_{\mathscr{F}_{0}}^{b}\left([-\infty, 0], R^{n}\right)$ is the family of all $\mathscr{F}_{0}$-measurable $C\left([-\infty, 0] ; R^{n}\right)$-valued random variables satisfying $\sup _{-\infty \leq s \leq 0} E\|\varphi(s)\|^{2}<\infty$, in which $E$ denotes expectations with respect to $\mathscr{P}$ and $C([-\infty, 0])$ denotes the family of all continuous $R^{n}$-valued functions $\varphi(s)$ on $[-\infty, 0]$.

We can describe the switched stochastic Cohen-Grossberg neural networks as follows:

$$
\begin{aligned}
& d x(t)=-\widehat{\alpha}(x(t))\left[\hat{\beta}(x(t))-A_{\sigma(t)} F(x(t))\right. \\
& -B_{\sigma(t)} F(x(t-\tau(t))) \\
& \left.\quad-C_{\sigma(t)} \int_{-\infty}^{t} K(t-s) F(x(s)) d s-J\right] d t \\
& +\sigma(t, x(t), x(t-\tau(t))) d \omega(t),
\end{aligned}
$$

where the function $\sigma(t):[0,+\infty) \rightarrow N=\{1,2, \ldots, N\}$ is the switching signal, which is deterministic, piecewise constant, and right continuous.

To continue our discussion, we give the following basic assumptions.

(H1) We assume there exist constants $\delta_{j}^{-}$and $\delta_{j}^{+}, i=1$, $2, \ldots, n$, such that

$$
\delta_{j}^{-} \leq \frac{f_{j}(x)-f_{j}(y)}{x-y} \leq \delta_{j}^{+}, \quad \forall x, y \in R, x \neq y .
$$

(H2) There exist positive constants $\underline{\alpha}_{i}, \bar{\alpha}_{i}$, for all $i=1$, $2, \ldots, n$, such that

$$
\underline{\alpha}_{i} \leq a_{i}\left(x_{i}(t)\right) \leq \bar{\alpha}_{i} .
$$

(H3) There exist positive constants $b_{j}$, such that

$$
x_{j}(t) \widehat{\beta}_{j}\left(x_{j}(t)\right) \geq b_{j} x_{j}^{2}(t) .
$$

(H4) We assume that the stochastic term satisfies $\sigma(t, \cdot, \cdot)$ : $R^{+} \times R^{n} \times R^{n} \rightarrow R^{n \times m}(\sigma(t, 0,0)=0)$ which is locally Lipschitz continuous and satisfies the linear growth condition as well. Moreover, $\sigma(t, \cdot, \cdot)$ satisfies the following condition:

$$
\begin{array}{r}
\operatorname{trace}\left[\sigma^{T}(t, x, y) \sigma(t, x, y)\right] \\
\leq x^{T} \Pi_{1}^{T} \Pi_{1} x+y^{T} \Pi_{2}^{T} \Pi_{2} y,
\end{array}
$$

where $\Pi_{i},(i=1,2)$ are known constant matrices with appropriate dimensions. 
(H5) The $\Delta A_{i}(t), \Delta B_{i}(t)$, and $\Delta C_{i}(t)$ are unknown matrices that represent the time-varying parameter uncertainties and are assumed to be of the following form:

$$
\begin{gathered}
\Delta A_{i}(t)=H_{A_{i}} G_{A_{i}}(t) E_{A_{i}}, \\
\Delta B_{i}(t)=H_{B_{i}} G_{B_{i}}(t) E_{B_{i}}, \\
\Delta C_{i}(t)=H_{C_{i}} G_{C_{i}}(t) E_{C_{i}},
\end{gathered}
$$

where $H_{A_{i}}, H_{B_{i}}, H_{C_{i}}, E_{A_{i}}, E_{B_{i}}$, and $E_{C_{i}}$ are known real constant matrices with appropriate dimensions. $G_{A_{i}}, G_{B_{i}}$, and $G_{C_{i}}$ may be time-varying matrices with Lebesgue measurable elements bounded by

$$
\begin{gathered}
G_{A_{i}}^{T}(t) G_{A_{i}} \leq I, \\
G_{B_{i}}^{T}(t) G_{B_{i}} \leq I, \\
G_{C_{i}}^{T}(t) G_{C_{i}} \leq I .
\end{gathered}
$$

Remark 1. The constants $l_{j}$ and $L_{j}$ can be positive, negative, or zero. Therefore, the activation functions $f(\cdot)$ are more general than the forms $\left|f_{j}(u)\right| \leq K_{j}|u|, K_{j}>0, j=1,2, \ldots, n$.

Remark 2. It is worth mentioning that the structures of the parametric uncertainties with the form (13) and (14) are more general than those in previous literature in $[28,34,37]$. However, $H_{A_{i}}=H_{B_{i}}=H_{C_{i}}$ has been discussed [28, 34, 37]. Recently, in [35], the attractor and boundedness of stochastic Cohen-Grossberg neural networks without parametric uncertainties were investigated.

Definition 3 (see [38]). The system (3) is mean-square uniformly ultimately bounded, if there exists a constant vector $\widetilde{B}>0$, for any constant $\varrho>0$; there is $t^{\prime}=t^{\prime}(\varrho)>0$, for all $t \geq t_{0}+t^{\prime}, t_{0}>0,\|\varphi\|<\varrho$; the solution $x\left(t, t_{0}, \varphi\right)$ of system (3) satisfies such that

$$
E\left\|x\left(t, t_{0}, \varphi\right)\right\|<\widetilde{B}
$$

where $E\left\|x\left(t, t_{0}, \varphi\right)\right\|=\sup _{-\infty \leq s \leq 0} E\left\|x_{i}\left(t+s, t_{0}, \varphi\right)\right\|$ and $\varphi \in$ $L_{\mathscr{F}_{0}}^{b}\left([-\infty, 0] ; R^{n}\right)$.

In this case, the set $\mathbb{A}=\left\{\varphi \in L_{\mathscr{F}_{0}}^{b} \mid E\|\varphi(s)\|_{\infty} \leq \widetilde{B}\right\}$ is called the attractor for the solution $x(t ; \varphi)$ of system (3) in the mean-square sense. Clearly, the proposition above is equal to

$$
\lim _{t \rightarrow \infty} \operatorname{supinf}_{y \in \mathbb{A}}\|x-y\|=0 .
$$

Definition 4 (see [39]). For the switching signal $\sigma(t)$, construct a switching sequence $\left\{\left(i_{0}, \sigma\left(t_{0}\right)\right), \ldots,\left(i_{k}, \sigma\left(t_{k}\right)\right), \ldots\right\}$, $\sigma\left(i_{k}\right) \in \underline{N}, k=0,1, \ldots, N$, where $t_{0}=0$ is the initial time and $t_{k}$ denotes the $i_{k}$ th switching instant. Moreover, $\sigma(t)=$ $i$ means that the $i$ th subsystem is activated. $N$ denotes the number of the subsystems. For each $T>t \geq 0$, let $N_{\sigma}(t, T)$ denote the number of discontinuities of $\sigma(t)$ in the interval $(t, T)$. If there exist $N_{0}>0$ and $T_{a}>0$ such that $N_{\sigma}(t, T) \leq$ $N_{0}+(T-t) / T_{a}$ holds, then $T_{a}$ is called the average dwell time. $N_{0}$ is the chatter bound.
Remark 5. It should be pointed out that for the chatter bound $N_{0}$, in our work, we take $N_{0} \geq 1$, which is more preferable than those previously reported in $[31,37]$. If $T_{a}=0$ is equivalent to the existence of a common function for all subsystems, this implies that switching signals can be arbitrary. Hence, the results reported in this paper are more effective than the arbitrary switching signals reported in the previous literatures $[28,37]$.

So, to obtain the main results of this paper, we introduce the following lemmas.

Lemma 6 (see [40]). For any positive-definite constant matrix $Z \in R^{n \times n}$, scalar $\tau>0$, and vector function $u(t):[t-r, t] \rightarrow$ $R^{n}, t \geq 0$, then

\section{(1) Jensen's inequality}

$$
\begin{aligned}
& -\int_{t-\tau}^{t} \varrho^{T}(s) Z \varrho(s) d s \\
& \quad \leq-\frac{1}{\tau}\left(\int_{t-\tau}^{t} \varrho(s) d s\right)^{T} Z\left(\int_{t-\tau}^{t} \varrho(s) d s\right),
\end{aligned}
$$

(2)

$$
\begin{aligned}
& -\int_{\beta}^{\alpha} \int_{t+\theta}^{t} \varrho^{T}(s) Z \varrho(s) d s d \theta \\
& \leq-\frac{2}{\alpha^{2}-\beta^{2}}\left(\int_{\beta}^{\alpha} \int_{t+\theta}^{t} \varrho(s) d s d \theta\right)^{T} \\
& \cdot Z\left(\int_{\beta}^{\alpha} \int_{t+\theta}^{t} \varrho(s) d s d \theta\right) .
\end{aligned}
$$

Lemma 7 (see [41]). Let $\mathscr{L}, \mathscr{M}_{1}$, and $\mathscr{M}_{2}$ be real matrices of appropriate dimension such that $\mathscr{L}^{T} \mathscr{L} \leq I$. Then for any scalar $\varepsilon>0$ and vectors $x$ and $y$ with appropriate dimensions, the following inequality is true:

$$
2 \mathscr{M}_{1}^{T} \mathscr{L} \mathscr{M}_{2} y \leq \varepsilon^{-1} x^{T} \mathscr{M}_{1} \mathscr{M}_{1}^{T} x+\varepsilon y^{T} \mathscr{M}_{2}^{T} \mathscr{M} y .
$$

Lemma 8 (see [41]). For any real matrix $X, Y$ and one positive-definite matrix $G$, the following matrix inequality holds:

$$
2 X^{T} G Y \leq \varepsilon X^{T} G X+\varepsilon^{-1} Y^{T} G Y
$$

Lemma 9 (see [42], Schur's complement). The LMI

$$
\Omega=\left(\begin{array}{cc}
\Omega_{11} & \Omega_{12} \\
* & \Omega_{22}
\end{array}\right)<0
$$

with $\Omega_{11}=\Omega_{11}^{T}, \Omega_{22}=\Omega_{22}^{T}$ is equivalent to

$$
\begin{aligned}
\Omega_{22} & <0, \\
\Omega_{11}-\Omega_{12} \Omega_{22}^{-1} \Omega_{12}^{T} & <0 .
\end{aligned}
$$




\section{Main Results}

Theorem 10. For given constants $\beta>0, \varepsilon>0, \mu<1$, if there exist positive scalars $\lambda$ and positive-definite matrices $P=\operatorname{diag}\left(p_{1}, p_{2}, \ldots, p_{n}\right), L=\operatorname{diag}\left(l_{1}, l_{2}, \ldots, l_{n}\right), D_{i}=$ $\operatorname{diag}\left(D_{i 1}, D_{i 2}, \ldots, D_{i n}\right),(i=1,2), Q_{1}, Q_{2}, Q_{3}, Q_{4}, R, S, \widetilde{Z}, Z$, $U$, such that the following conditions hold:

$\Delta_{1}$

$=\left[\begin{array}{cccccccccccc}\phi_{11} & 0 & 0 & 0 & \phi_{15} & 0 & 0 & \phi_{18} & \phi_{19} & 0 & 0 & 0 \\ * & \phi_{22} & 0 & 0 & 0 & \phi_{26} & 0 & \phi_{28} & 0 & 0 & 0 & 0 \\ * & * & \phi_{33} & 0 & 0 & 0 & \phi_{37} & 0 & \phi_{39} & 0 & 0 & 0 \\ * & * & * & \phi_{44} & \phi_{45} & 0 & 0 & \phi_{48} & \phi_{49} & 0 & 0 & 0 \\ * & * & * & * & \phi_{55} & 0 & 0 & 0 & 0 & 0 & 0 & 0 \\ * & * & * & * & * & \phi_{66} & 0 & 0 & 0 & 0 & 0 & 0 \\ * & * & * & * & * & * & \phi_{77} & 0 & 0 & 0 & 0 & 0 \\ * & * & * & * & * & * & * & \phi_{88} & 0 & 0 & 0 & 0 \\ * & * & * & * & * & * & * & * & \phi_{99} & 0 & 0 & 0 \\ * & * & * & * & * & * & * & * & * & \phi_{10} & 0 & 0 \\ * & * & * & * & * & * & * & * & * & * & \bar{\phi}_{11} & 0 \\ * & * & * & * & * & * & * & * & * & * & * & \bar{\phi}_{12}\end{array}\right]$

$<0$,

$P<\lambda I$

where

$$
\begin{aligned}
\phi_{11} & =\frac{1}{\bar{a}^{2}}\left[\beta P-2 \Omega_{1} P \Omega_{2}+\lambda \Pi_{1}^{T} \Pi_{1}+\varepsilon \Omega_{4} D_{1}+P\right. \\
& +\frac{1}{4 \beta^{2}} I+\tau_{M} e^{\beta \tau_{M}} Q_{1}+\tau_{m} e^{\beta \tau_{m}} Q_{2}+e^{\beta \tau_{M}} Q_{3}+\tau_{M} R \\
& \left.+\left(\tau_{M}-\tau_{m}\right) S+\frac{\tau_{M}^{2}}{4} Z+\frac{\tau_{M}^{2}}{4} \widetilde{Z}-\Omega_{3} D_{1}\right], \\
\phi_{15} & =P A, \\
\phi_{18} & =P B, \\
\phi_{19} & =P C, \\
\phi_{22} & =-\tau_{M} Q_{1}, \\
\phi_{26} & =\phi_{28}=0, \\
\phi_{33} & =-\tau_{m} Q_{2}, \\
\phi_{37} & =\phi_{39}=0, \\
\phi_{44} & =\lambda \Pi_{2}^{T} \Pi_{2}-\Omega_{3} D_{2}+\frac{1}{4 \beta^{2}} I-(1-\mu) Q_{3}, \\
\phi_{45} & =0, \\
\phi_{48} & =\Omega_{4} D_{2}, \\
\phi_{49} & =0,
\end{aligned}
$$

$$
\begin{aligned}
& \phi_{55}=e^{\beta \tau_{M}} Q_{4}+L K(\iota)+\varepsilon^{-1} \Omega_{4} D_{1}+\tau_{M}^{2} U-D_{1}+\frac{1}{\beta^{2}} \\
& I, \\
& \phi_{66}=-\frac{e^{-\beta \tau_{M}}}{\tau_{M}} R, \\
& \phi_{77}=-\frac{e^{-\beta \tau_{M}}}{\tau_{M}-\tau_{m}} S, \\
& \phi_{88}=-(1-\mu) Q_{4}-D_{2}+\frac{1}{\beta^{2}} I, \\
& \phi_{99}=-L, \\
& \phi_{10}=-(1-\mu) e^{-\beta \tau_{M}} U, \\
& \bar{\phi}_{11}=-e^{-\beta \tau_{M}} Z, \\
& \bar{\phi}_{12}=-e^{-\beta \tau_{M}} \widetilde{Z}, \\
& D_{i}>0, \quad i=1,2, \operatorname{diag}\left\{\delta_{1}^{-} \delta_{1}^{+}, \delta_{2}^{-} \delta_{2}^{+}, \ldots, \delta_{n}^{-} \delta_{n}^{+}\right\}, \\
& \Omega_{3}=\operatorname{diag}\left\{\frac{\delta_{1}^{-}+\delta_{1}^{+}}{2}, \frac{\delta_{2}^{-}+\delta_{2}^{+}}{2}, \ldots, \frac{\delta_{n}^{-}+\delta_{n}^{+}}{2}\right\}, \\
&\left.\Omega_{4}\right\}
\end{aligned}
$$

then system (3) is mean-square uniformly ultimately bounded.

Proof. Choose the following Lyapunov-Krasovskii functional:

$$
V(t)=V_{1}(t)+V_{2}(t)+V_{3}(t)+V_{4}(t)+V_{5}(t)
$$

where

$$
\begin{aligned}
& V_{1}(t)=e^{\beta t} x^{T}(t) P x(t) \\
& V_{2}(t)=\tau_{M} \int_{t-\tau_{M}}^{t} e^{\beta\left(s+\tau_{M}\right)} x^{T}(s) Q_{1} x(s) d s \\
& \quad+\tau_{m} \int_{t-\tau_{m}}^{t} e^{\beta\left(s+\tau_{m}\right)} x^{T}(s) Q_{2} x(s) d s \\
& \quad+\int_{t-\tau(t)}^{t} e^{\beta\left(s+\tau_{M}\right)} x^{T}(s) Q_{3} x(s) d s \\
& \quad+\int_{t-\tau(t)}^{t} e^{\beta\left(s+\tau_{M}\right)} F^{T}(x(s)) Q_{4} F(x(s)) d s, \\
& V_{3}(t)=\int_{-\tau_{M}}^{0} \int_{t+\theta}^{t} e^{\beta s} x^{T}(s) R x(s) d s d \theta \\
& \quad+\int_{-\tau_{M}}^{-\tau_{m}} \int_{t+\theta}^{t} e^{\beta s} x^{T}(s) S x(s) d s d \theta \\
& \quad+\tau_{M} \int_{-\tau(t)}^{0} \int_{t+\theta}^{t} e^{\beta s} F^{T}(x(s)) U F(x(s)) d s d \theta
\end{aligned}
$$




$$
\begin{aligned}
& V_{4}(t)=\frac{\tau_{M}^{2}}{2} \int_{-\tau_{M}}^{0} \int_{\theta}^{0} \int_{t+\lambda}^{t} e^{\beta s} x^{T}(s) Z x(s) d s d \lambda d \theta \\
& +\frac{\left(\tau_{M}^{2}-\tau_{m}^{2}\right)}{2} \\
& \quad \cdot \int_{-\tau_{M}}^{-\tau_{m}} \int_{\theta}^{0} \int_{t+\lambda}^{t} e^{\beta s} x^{T}(s) \widetilde{Z} X(s) d s d \lambda d \theta, \\
& V_{5}(t)=\sum_{j=1}^{n} l_{j} \int_{0}^{\infty} k_{j}(\delta) \int_{t-\delta}^{t} e^{\beta(\gamma+\delta)} F_{j}^{2}\left(x_{j}(\gamma)\right) d \gamma d \delta .
\end{aligned}
$$

By applying the Itô differential formula, the stochastic derivative of $V(t)$ along the trajectory of system (3) is

$$
\begin{aligned}
d V(t) & \\
= & \mathscr{L} V d t \\
& +2 e^{\beta t} x^{T}(t) P \sigma(t, x(t), x(t-\tau(t))) d \omega(t) .
\end{aligned}
$$

With the infinitesimal-operator, we can deduce that

$$
\begin{aligned}
& \mathscr{L} V_{1}=\beta e^{\beta t} x^{T}(t) P x(t)-2 e^{\beta t} x^{T}(t) P \alpha(x(t)) \\
& \cdot[\beta(x(t))-A F(x(t)) \\
& -C \int_{-\infty}^{t} K(t-s) F(x(s)) d s-B F(x(t-\tau(t))) \\
& -J]+e^{\beta t} \frac{1}{2} \operatorname{tra}\left[\sigma(t, x(t), x(t-\tau(t)))^{T} V_{1 x x}(x, t)\right. \\
& \cdot \sigma(t, x(t), x(t-\tau(t)))] .
\end{aligned}
$$

Denote $\Omega_{1}=\operatorname{diag}\left\{\underline{\alpha}_{1}, \underline{\alpha}_{2}, \ldots, \underline{\alpha}_{n}\right\}, \Omega_{2}=\operatorname{diag}\left\{b_{1}, b_{2}, \ldots, b_{n}\right\}$, according to assumptions ( $\mathrm{H} 2)$ and (H3). Then, we obtain the following inequalities:

$$
\begin{aligned}
- & 2 e^{\beta t} x^{T}(t) P \alpha(x(t)) \beta(x(t)) \\
& =-2 e^{\beta t} \sum_{j=1}^{n} x_{j}(t) p_{j} \alpha_{j}\left(x_{j}(t)\right) \beta_{j}\left(x_{j}(t)\right) \\
\leq & -2 e^{\beta t} \sum_{j=1}^{n} \underline{\alpha}_{j} p_{j} b_{j} x_{j}^{2}(t)=-2 e^{\beta t} x^{T}(t) \Omega_{1} P \Omega_{2} x(t) .
\end{aligned}
$$

By assumption (H4), we have

$$
\begin{gathered}
\frac{1}{2} e^{\beta t} \operatorname{tra}\left[\sigma(t, x(t), x(t-\tau(t)))^{T} V_{1 x x}(x, t)\right. \\
\cdot \sigma(t, x(t), x(t-\tau(t)))]=e^{\beta t} \lambda_{\max }(P) \\
\cdot \operatorname{tra}\left[\sigma(t, x(t), x(t-\tau(t)))^{T}\right. \\
\cdot \sigma(t, x(t), x(t-\tau(t)))] \leq \lambda e^{\beta t} x^{T}(t) \Pi_{1}^{T} \Pi_{1} x(t) \\
+\lambda e^{\beta t} x^{T}(t-\tau(t)) \Pi_{2}^{T} \Pi_{2} x(t-\tau(t)) .
\end{gathered}
$$

Combining inequalities (29) and (30), we derive

$$
\begin{aligned}
\mathscr{L} V_{1} & =\beta e^{\beta t} x^{T}(t) P x(t)-2 e^{\beta t} x^{T}(t) \Omega_{1} P \Omega_{2} x(t) \\
+ & e^{\beta t}\left[2 x^{T}(t) P \alpha(x(t)) A F(x(t))\right. \\
& +2 x^{T}(t) P \alpha(x(t)) B F(x(t-\tau(t))) \\
& +2 x^{T}(t) P \alpha(x(t)) C \int_{-\infty}^{t} K(t-s) F(x(s)) \\
& \left.+2 x^{T}(t) P \alpha(x(t)) J\right]+\rho_{1}\left[x^{T}(t) \Pi_{1}^{T} P \Pi_{1} x(t)\right] \\
+ & \rho_{2} e^{\beta t} x^{T}(t-\tau(t)) \Pi_{2}^{T} P \Pi_{2} x(t-\tau(t)) \\
& \leq e^{\beta t}\left[\beta x^{T}(t) P x(t)-2 x^{T}(t) \Omega_{1} P \Omega_{2} x(t)\right] \\
+ & {\left[2 x^{T}(t) P \alpha(x(t)) A F(x(t))\right.} \\
+ & +e^{\beta t}\left[\lambda x^{T}(t) \Pi_{1}^{T} \Pi_{1} x(t) P \alpha(x(t)) B F(x(t-\tau(t)))\right. \\
+ & 2 x^{T}(t) P \alpha(x(t)) C \int_{-\infty}^{t} K(t-s) F(x(s)) \\
+ & \left.x^{T}(t) P x(t)+\bar{\alpha}^{2} J^{T} P J\right] e^{\beta t}
\end{aligned}
$$

where $\bar{\alpha}=\max \left\{\bar{\alpha}_{1}, \bar{\alpha}_{2}, \ldots, \bar{\alpha}_{n}\right\}$.

Then, we easily derive

$$
\begin{aligned}
\mathscr{L} & V_{2}=\tau_{M} e^{\beta\left(t+\tau_{M}\right)} x^{T}(t) Q_{1} x(t)-\tau_{M} e^{\beta t} x^{T}\left(t-\tau_{M}\right) \\
& Q_{1} x\left(t-\tau_{M}\right)+\tau_{m} e^{\beta\left(t+\tau_{m}\right)} x^{T}(t) Q_{2} x(t) \\
& -\tau_{m} e^{\beta t} x^{T}\left(t-\tau_{m}\right) Q_{2} x\left(t-\tau_{m}\right) \\
& +e^{\beta\left(t+\tau_{M}\right)}\left[x^{T}(t) Q_{3} x(t)+F^{T}(x(t)) Q_{4} F(x(t))\right] \\
& -(1-\dot{\tau}(t)) e^{\beta\left(t-\tau(t)+\tau_{M}\right)}\left[x^{T}(t-\tau(t)) Q_{3} x(t \tau(t))\right. \\
& \left.+F^{T}(x(t-\tau(t))) Q_{4} F(x(t-\tau(t)))\right] \\
& \leq \tau_{M} e^{\beta\left(t+\tau_{M}\right)} x^{T}(t) Q_{1} x(t)-\tau_{M} e^{\beta t} x^{T}\left(t-\tau_{M}\right) \\
& +Q_{1} x\left(t-\tau_{M}\right)+\tau_{m} e^{\beta\left(t+\tau_{m}\right)} x^{T}(t) Q_{2} x(t) \\
& -\tau_{m} e^{\beta t} x^{T}\left(t-\tau_{m}\right) Q_{2} x\left(t-\tau_{m}\right) \\
& +e^{\beta\left(t+\tau_{M}\right)}\left[x^{T}(t) Q_{3} x(t)+F^{T}(x(t)) Q_{4} F(x(t))\right] \\
& -(1-\mu) e^{\beta t}\left[x^{T}(t-\tau(t)) Q_{3} x(t \tau(t))\right. \\
& \left.+F^{T}(x(t-\tau(t))) Q_{4} F(x(t-\tau(t)))\right] .
\end{aligned}
$$


Similarly, calculating the operation of $\mathscr{L} V_{3}$ along the trajectory of system (3), one can get

$$
\begin{aligned}
& \mathscr{L} V_{3}=\tau_{M} e^{\beta t} x^{T}(t) R x(t)-\int_{t-\tau_{M}}^{t} e^{\beta s} x^{T}(s) R x(s) d s \\
& +\left(\tau_{M}-\tau_{m}\right) e^{\beta t} x^{T}(t) R x(t) \\
& -\int_{t-\tau_{M}}^{t-\tau_{m}} e^{\beta s} x^{T}(s) R x(s) d s+\tau_{M} \tau(t) e^{\beta t} F^{T}(x(t)) \\
& \cdot U F(x(t))-\tau_{M}(1-\dot{\tau}(t)) \\
& \cdot \int_{t-\tau(t)}^{t} e^{\beta(t-\tau(t))} F^{T}(x(s)) U F(x(s)) d s \\
& \leq \tau_{M} e^{\beta t} x^{T}(t) R x(t) \\
& -e^{\beta\left(t-\tau_{M}\right)} \int_{t-\tau_{M}}^{t} x^{T}(s) R x(s) d s+\left(\tau_{M}-\tau_{m}\right) \\
& \cdot e^{\beta t} x^{T}(t) R x(t)-e^{\beta\left(t-\tau_{M}\right)} \int_{t-\tau_{M}}^{t-\tau_{m}} x^{T}(s) R s(t) d s \\
& +\tau_{M}^{2} e^{\beta t} F^{T}(x(t)) U F(x(t))-\tau_{M}(1-\mu) \\
& \cdot e^{\beta\left(t-\tau_{M}\right)} \int_{t-\tau(t)}^{t} F^{T}(x(s)) U F(x(s)) d s .
\end{aligned}
$$

By Lemma 6 (1), it follows that

$$
\begin{aligned}
& -e^{-\beta \tau_{M}} \int_{t-\tau_{M}}^{t} x^{T}(s) R x(s) d s \\
& \leq-\frac{e^{-\beta \tau_{M}}}{\tau_{M}}\left(\int_{t-\tau_{M}}^{t} x(s) d s\right)^{T} R\left(\int_{t-\tau_{M}}^{t} x(s) d s\right) \\
& -e^{-\beta \tau_{M}} \int_{t-\tau_{M}}^{t-\tau_{m}} x^{T}(s) S x(s) d s \\
& \leq-\frac{e^{-\beta \tau_{M}}}{\tau_{M}-\tau_{m}}\left(\int_{t-\tau_{M}}^{t-\tau_{m}} x(s) d s\right)^{T} S\left(\int_{t-\tau_{M}}^{t-\tau_{m}} x(s) d s\right) \\
& -\tau_{M} e^{-\beta \tau_{M}} \int_{t-\tau(t)}^{t} F^{T}(x(s)) U F(x(s)) d s \\
& \leq-e^{-\beta \tau_{M}}\left(\int_{t-\tau(t)}^{t} F(x(s)) d s\right)^{T} \\
& \cdot U\left(\int_{t-\tau(t)}^{t} F(x(s)) d s\right) .
\end{aligned}
$$

Similarly, one can derive

$$
\begin{aligned}
& \mathscr{L} V_{4}=\frac{\tau_{M}^{2}}{4} e^{\beta t} x^{T}(t) Z x(t)-\frac{\tau_{M}^{2}}{2} \\
& \cdot \int_{-\tau_{M}}^{0} \int_{t+\theta}^{t} e^{\beta s} x^{T}(t) Z x(t) d s d \theta+\frac{\left(\tau_{M}^{2}-\tau_{m}^{2}\right)}{4} \\
& \cdot e^{\beta t} x^{T}(t) \widetilde{Z} x(t)-\frac{\left(\tau_{M}^{2}-\tau_{m}^{2}\right)}{2}
\end{aligned}
$$

$$
\begin{aligned}
& \int_{-\tau_{M}}^{-\tau_{m}} \int_{t+\theta}^{t} e^{\beta s} x^{T}(t) \widetilde{Z} x(t) d s d \theta \\
& \leq e^{\beta t}\left[\frac{\tau_{M}^{2}}{4} x^{T}(t) Z x(t)\right. \\
& -\frac{e^{-\beta \tau_{M}} \tau_{M}^{2}}{2} \int_{-\tau_{M}}^{0} \int_{t+\theta}^{t} x^{T}(t) Z x(t) d s d \theta \\
& +\frac{\left(\tau_{M}^{2}-\tau_{m}^{2}\right)}{4} x^{T}(t) \widetilde{Z} x(t) \\
& \left.-\frac{e^{-\beta \tau_{M}}\left(\tau_{M}^{2}-\tau_{m}^{2}\right)}{2} \int_{-\tau_{M}}^{-\tau_{m}} \int_{t+\theta}^{t} x^{T}(t) \widetilde{Z} x(t) d s d \theta\right] .
\end{aligned}
$$

Using Lemma 6 (2), we have

$$
\begin{gathered}
-\frac{e^{-\beta \tau_{M}} \tau_{M}^{2}}{2} \int_{-\tau_{M}}^{0} \int_{t+\theta}^{t} x^{T}(t) Z x(t) d s d \theta \\
\leq-e^{-\beta \tau_{M}}\left(\int_{-\tau_{M}}^{0} \int_{t+\theta}^{t} x(s) d s d \theta\right)^{T} \\
\cdot Z\left(\int_{-\tau_{M}}^{0} \int_{t+\theta}^{t} x(s) d s d \theta\right) .
\end{gathered}
$$

Similarly, one can obtain

$$
\begin{aligned}
& -\frac{e^{-\beta \tau_{M}}\left(\tau_{M}^{2}-\tau_{m}^{2}\right)}{2} \int_{-\tau_{M}}^{-\tau_{m}} \int_{t+\theta}^{t} x^{T}(t) \widetilde{Z} x(t) d s d \theta \\
& \quad \leq-e^{-\beta \tau_{M}}\left(\int_{-\tau_{M}}^{-\tau_{m}} \int_{t+\theta}^{t} x(t) d s d \theta\right)^{T} \\
& \quad \cdot \widetilde{Z}\left(\int_{-\tau_{M}}^{-\tau_{m}} \int_{t+\theta}^{t} x(t) d s d \theta\right) .
\end{aligned}
$$

Then, noting the condition $\int_{0}^{\infty} k_{j}(s) d s=1$ and the CauchySchwarz inequality

$$
\left(\int p(s) q(s) d s\right)^{2} \leq\left(\int p^{2}(s) d s\right)\left(\int q^{2}(s) d s\right),
$$

it can yield

$$
\begin{gathered}
\mathscr{L} V_{5}=\sum_{j=1}^{n} l_{j} \int_{0}^{\infty} k_{j}(\delta) e^{\beta(t+\delta)} F_{j}^{2}\left(x_{j}(t)\right) d \delta-\sum_{j=1}^{n} l_{j} \\
\cdot \int_{0}^{\infty} k_{j}(\delta) e^{\beta t} F_{j}^{2}\left(x_{j}(t-\delta)\right) d \delta
\end{gathered}
$$




$$
\begin{aligned}
& =e^{\beta t} \sum_{j=1}^{n} l_{j} \bar{k}_{j}(\iota) F_{j}^{2}\left(x_{j}(t)\right) d \delta-e^{\beta t} \sum_{j=1}^{n} l_{j} \\
& \cdot \int_{0}^{\infty} k_{j}(\delta) d \delta \int_{0}^{\infty} k_{j}(\delta) F_{j}^{2}\left(x_{j}(t-\delta)\right) d \delta \\
& \leq e^{\beta t} F^{T}(x(t)) L K(\iota) F(x(t))-\sum_{j=1}^{n} l_{j} \\
& \cdot\left(\int_{0}^{\infty} k_{j}(\delta) F_{j}\left(x_{j}(t-\delta)\right) d \delta\right)^{2}=e^{\beta t} F^{T}(x(t)) \\
& \cdot L K(\iota) F(x(t))-\left(\int_{-\infty}^{t} K(t-s) F(x(s)) d s\right)^{T} \\
& \cdot L\left(\int_{-\infty}^{t} K(t-s) F(x(s)) d s\right) .
\end{aligned}
$$

Then, based on assumption (H1), it easy to see that, for $j=1,2, \ldots, n$,

$$
\begin{aligned}
& {\left[F_{j}\left(x_{j}(t)\right)-F_{j}(0)-\delta_{j}^{-} x_{j}(t)\right]} \\
& \cdot\left[F_{j}\left(x_{j}(t)\right)-F_{j}(0)-\delta_{j}^{+} x_{j}(t)\right] \leq 0, \\
& {\left[F_{j}\left(x_{j}(t-\tau(t))\right)-F_{j}(0)-\delta_{j}^{-} x_{j}(t-\tau(t))\right]} \\
& \cdot\left[F_{j}\left(x_{j}(t-\tau(t))\right)-F_{j}(0)-\delta_{j}^{+} x_{j}(t-\tau(t))\right] \\
& \quad \leq 0 .
\end{aligned}
$$

Then, let

$$
\begin{aligned}
\Upsilon_{1} & =-\sum_{j=1}^{n} D_{1 j}\left[F_{j}\left(x_{j}(t)\right)-F_{j}(0)-\delta_{j}^{-} x_{j}(t)\right] \\
& \cdot\left[F_{j}\left(x_{j}(t)\right)-F_{j}(0)-\delta_{j}^{+} x_{j}(t)\right] \geq 0, \\
\Upsilon_{2} & =-\sum_{j=1}^{n} D_{2 j}\left[F_{j}\left(x_{j}(t-\tau(t))\right)-F_{j}(0)\right. \\
& \left.-\delta_{j}^{-} x_{j}(t-\tau(t))\right]\left[F_{j}\left(x_{j}(t-\tau(t))\right)-F_{j}(0)\right. \\
& \left.-\delta_{j}^{+} x_{j}(t-\tau(t))\right] \geq 0 .
\end{aligned}
$$

Therefore,

$$
\begin{aligned}
\Upsilon_{1} & +\Upsilon_{2}=-\sum_{j=1}^{n} D_{1 j}\left[F_{j}\left(x_{j}(t)\right)-F_{j}(0)-\delta_{j}^{-} x_{j}(t)\right] \\
& \cdot\left[F_{j}\left(x_{j}(t)\right)-F_{j}(0)-\delta_{j}^{+} x_{j}(t)\right] \\
& -\sum_{j=1}^{n} D_{2 j}\left[F_{j}\left(x_{j}(t-\tau(t))\right)-F_{j}(0)\right. \\
- & \left.\delta_{j}^{-} x_{j}(t-\tau(t))\right]\left[F_{j}\left(x_{j}(t-\tau(t))\right)-F_{j}(0)\right. \\
- & \left.\delta_{j}^{+} x_{j}(t-\tau(t))\right]=-\sum_{j=1}^{n} D_{1 j}\left[F_{j}\left(x_{j}(t)\right)\right.
\end{aligned}
$$

$$
\begin{aligned}
& \left.-\delta_{j}^{-} x_{j}(t)\right]\left[F_{j}\left(x_{j}(t)\right)-\delta_{j}^{+} x_{j}(t)\right]-\sum_{j=1}^{n} D_{2 j} \\
& \cdot\left[F_{j}\left(x_{j}(t-\tau(t))\right)-\delta_{j}^{-} x_{j}(t-\tau(t))\right] \\
& \cdot\left[F_{j}\left(x_{j}(t-\tau)\right)-\delta_{j}^{+} x_{j}(t-\tau(t))\right]-\sum_{j=1}^{n} D_{1 j} F_{j}^{2}(0) \\
& +\sum_{j=1}^{n} D_{1 j} F_{j}(0)\left[2 F_{j}\left(x_{j}(t)\right)-\left(\delta_{j}^{+}+\delta_{j}^{-}\right) x_{j}(t)\right] \\
& -\sum_{j=1}^{n} D_{2 j} F_{j}^{2}(0)+\sum_{j=1}^{n} D_{2 j} F_{j}(0)\left[2 F_{j}\left(x_{j}(t-\tau(t))\right)\right. \\
& \left.-\left(\delta_{j}^{+}+\delta_{j}^{-}\right) x_{j}(t-\tau(t))\right] \leq-\sum_{j=1}^{n} D_{1 j}\left[F_{j}\left(x_{j}(t)\right)\right. \\
& \left.-\delta_{j}^{-} x_{j}(t)\right]\left[F_{j}\left(x_{j}(t)\right)-\delta_{j}^{+} x_{j}(t)\right]-\sum_{j=1}^{n} D_{2 j} \\
& \cdot\left[F_{j}\left(x_{j}(t-\tau(t))\right)-\delta_{j}^{-} x_{j}(t-\tau(t))\right] \\
& \cdot\left[F_{j}\left(x_{j}(t-\tau(t))\right)-\delta_{j}^{+} x_{j}(t-\tau(t))\right] \\
& +\sum_{j=1}^{n}\left[\frac{1}{\beta^{2}} F_{j}^{2}\left(x_{j}(t)\right)+\beta^{2} D_{1 j}^{2} F_{j}^{2}(0)+\frac{1}{4 \beta^{2}} x_{j}^{2}(t)\right. \\
& \left.+\beta^{2} D_{1 j}^{2} F_{j}^{2}(0)\left(\delta_{j}^{+}+\delta_{j}^{-}\right)^{2}\right] \\
& +\sum_{j=1}^{n}\left[\frac{1}{\beta^{2}} F_{j}^{2}\left(x_{j}(t-\tau(t))\right)+\beta^{2} D_{2 j}^{2} F_{j}^{2}(0)\right. \\
& \left.+\frac{1}{4 \beta^{2}} x_{j}^{2}(t-\tau(t))+\beta^{2} D_{2 j}^{2} F_{j}^{2}(0)\left(\delta_{j}^{+}+\delta_{j}^{-}\right)^{2}\right] .
\end{aligned}
$$

From Lemma 8, the following inequality holds true:

$$
\begin{aligned}
2 x^{T}(t) & \Omega_{4} D_{1} F(x(t)) \\
\leq & \varepsilon x^{T}(t) \Omega_{4} D_{1} x(t) \\
& +\varepsilon^{-1} F^{T}(x(t)) \Omega_{4} D_{1} F(x(t)) .
\end{aligned}
$$

\section{Denote}

$$
\begin{gathered}
\zeta^{T}(t)=\left[x^{T}(t), x^{T}\left(t-\tau_{M}\right), x^{T}\left(t-\tau_{m}\right),\right. \\
x^{T}(t-\tau(t)), F^{T}(x(t)),\left(\int_{t-\tau_{M}}^{t} x(s) d s\right)^{T}, \\
\left(\int_{t-\tau_{M}}^{t-\tau_{m}} x(s) d s\right)^{T}, F^{T}(x(t-\tau(t))),
\end{gathered}
$$




$$
\begin{aligned}
& \left(\int_{-\infty}^{t} K(t-s) F(x(s)) d s\right)^{T}, \\
& \left.\left(\int_{t-\tau(t)}^{t} F(x(s)) d s\right)^{T}, y_{1}^{T}(t), y_{2}^{T}(t)\right],
\end{aligned}
$$

where

$$
\begin{aligned}
& y_{1}(t)=\left(\int_{-\tau_{M}}^{0} \int_{t+\theta}^{t} x^{T}(s) Z x(s) d s d \theta\right), \\
& y_{2}(t)=\left(\int_{-\tau_{M}}^{-\tau_{m}} \int_{t+\theta}^{t} x^{T}(s) \widetilde{Z} x(s) d s d \theta\right) .
\end{aligned}
$$

Using (27)-(43), we can derive

$$
\begin{aligned}
& d V(t) \leq \mathscr{L} V d t+2 e^{\beta t} x^{T}(t) P \sigma(t, x(t), x(t \\
& -\tau(t))) d \omega(t)+e^{\beta t}\left(\Upsilon_{1}+\Upsilon_{2}\right) \leq e^{\beta t} \zeta^{T}(t) \\
& \cdot \Delta \zeta(t)+e^{\beta t} \bar{\alpha}^{2} J^{T} P J+2 e^{\beta t} x^{T}(t) P \sigma(t, x(t), x(t \\
& -\tau(t))) d \omega(t)+e^{\beta t} \sum_{j=1}^{n}\left[\beta^{2} D_{1 j}^{2} F_{j}^{2}(0)\right. \\
& +\beta^{2} D_{1 j}^{2} F_{j}^{2}(0)\left(\delta_{j}^{+}+\delta_{j}^{-}\right)^{2}+\beta^{2} D_{2 j}^{2} F_{j}^{2}(0) \\
& \left.+\beta^{2} D_{2 j}^{2} F_{j}^{2}(0)\left(\delta_{j}^{+}+\delta_{j}^{-}\right)^{2}\right],
\end{aligned}
$$

where

$$
\Delta=\left[\begin{array}{cccccccccccc}
\Phi_{11} & 0 & 0 & 0 & \Phi_{15} & 0 & 0 & \Phi_{18} & \Phi_{19} & 0 & 0 & 0 \\
* & \Phi_{22} & 0 & 0 & 0 & \Phi_{26} & 0 & \Phi_{28} & 0 & 0 & 0 & 0 \\
* & * & \Phi_{33} & 0 & 0 & 0 & \Phi_{37} & 0 & \Phi_{39} & 0 & 0 & 0 \\
* & * & * & \Phi_{44} & \Phi_{45} & 0 & 0 & \Phi_{48} & \Phi_{49} & 0 & 0 & 0 \\
* & * & * & * & \Phi_{55} & 0 & 0 & 0 & 0 & 0 & 0 & 0 \\
* & * & * & * & * & \Phi_{66} & 0 & 0 & 0 & 0 & 0 & 0 \\
* & * & * & * & * & * & \Phi_{77} & 0 & 0 & 0 & 0 & 0 \\
* & * & * & * & * & * & * & \Phi_{88} & 0 & 0 & 0 & 0 \\
* & * & * & * & * & * & * & * & \Phi_{99} & 0 & 0 & 0 \\
* & * & * & * & * & * & * & * & * & \Phi_{10} & 0 & 0 \\
* & * & * & * & * & * & * & * & * & * & \Phi_{11} & 0 \\
* & * & * & * & * & * & * & * & * & * & * & \Phi_{12}
\end{array}\right],
$$

$$
\begin{aligned}
\Phi_{11}= & \beta P-2 \Omega_{1} P \Omega_{2}+\lambda \Pi_{1}^{T} \Pi_{1}+\varepsilon \Omega_{4} D_{1}+P+\frac{1}{4 \beta^{2}} I+\tau_{M} e^{\beta \tau_{M}} Q_{1}+\tau_{m} e^{\beta \tau_{m}} Q_{2}+e^{\beta \tau_{M}} Q_{3}+\tau_{M} R+\left(\tau_{M}-\tau_{m}\right) S+\frac{\tau_{M}^{2}}{4} Z \\
& +\frac{\tau_{M}^{2}}{4} \widetilde{Z}-\Omega_{3} D_{1},
\end{aligned}
$$

$\Phi_{15}=P \alpha(x(t)) A$,

$\Phi_{18}=P \alpha(x(t)) B$,

$\Phi_{19}=P \alpha(x(t)) C$,

$\Phi_{22}=-\tau_{M} Q_{1}$,

$\Phi_{26}=\phi_{28}=0$,

$\Phi_{33}=-\tau_{m} Q_{2}$,

$\Phi_{37}=\Phi_{39}=0$,

$\Phi_{44}=\lambda \Pi_{2}^{T} \Pi_{2}-\Omega_{3} D_{2}+\frac{1}{4 \beta^{2}} I-(1-\mu) Q_{3}$,

$\Phi_{45}=0$, 


$$
\begin{aligned}
& \Phi_{48}=\Omega_{4} D_{2}, \\
& \Phi_{49}=0 \\
& \Phi_{55}=e^{\beta \tau_{M}} Q_{4}+L K(\iota)+\varepsilon^{-1} \Omega_{4} D_{1}+\tau_{M}^{2} U-D_{1}+\frac{1}{\beta^{2}} I, \\
& \Phi_{66}=-\frac{e^{-\beta \tau_{M}}}{\tau_{M}} R, \\
& \Phi_{77}=-\frac{e^{-\beta \tau_{M}}}{\tau_{M}-\tau_{m}} S, \\
& \Phi_{88}=-(1-\mu) Q_{4}-D_{2}+\frac{1}{\beta^{2}} I, \\
& \Phi_{99}=-L, \\
& \Phi_{10}=-(1-\mu) e^{-\beta \tau_{M}} U, \\
& \Phi_{11}=-e^{-\beta \tau_{M}} Z \\
& \bar{\Phi}_{12}=-e^{-\beta \tau_{M}} \widetilde{Z} .
\end{aligned}
$$

By integrating both sides of (46) in time interval $t \in\left[t_{0}, t\right]$ and taking expectation results in

$$
\begin{aligned}
& E\{V(x(t))\} \leq E\left\{V\left(x\left(t_{0}\right)\right)\right\}+E\left\{\beta^{-1} e^{\beta t} \bar{\alpha}^{2} J^{T} P J\right\} \\
& +E\left\{e ^ { \beta t } \sum _ { j = 1 } ^ { n } \left[\beta D_{1 j}^{2} F_{j}^{2}(0)+\beta D_{1 j}^{2} F_{j}^{2}(0)\left(\delta_{j}^{+}+\delta_{j}^{-}\right)^{2}\right.\right. \\
& \left.\left.+\beta D_{2 j}^{2} F_{j}^{2}(0)+\beta D_{2 j}^{2} F_{j}^{2}(0)\left(\delta_{j}^{+}+\delta_{j}^{-}\right)^{2}\right]\right\} .
\end{aligned}
$$

Note from (25) that

$$
E\{V(x(t))\} \geq \Upsilon E\|x(t)\|^{2} e^{\beta t},
$$

which implies

$$
E\|x(t)\|^{2} \leq \frac{e^{-\beta t} E\left\{V\left(x\left(t_{0}\right)\right)\right\}+\beta^{-1} \bar{\alpha}^{2} J^{T} P J+H}{\Upsilon},
$$

where $\Upsilon=\lambda_{\min }(P)$ and

$$
\begin{aligned}
H & =\sum_{j=1}^{n}\left[\beta D_{1 j}^{2} F_{j}^{2}(0)+\beta D_{1 j}^{2} F_{j}^{2}(0)\left(\delta_{j}^{+}+\delta_{j}^{-}\right)^{2}\right. \\
& \left.+\beta D_{2 j}^{2} F_{j}^{2}(0)+\beta D_{2 j}^{2} F_{j}^{2}(0)\left(\delta_{j}^{+}+\delta_{j}^{-}\right)^{2}\right] .
\end{aligned}
$$

If one chooses $\widetilde{B}=\sqrt{\left(1+\beta^{-1} \bar{\alpha}^{2} J^{T} P J+H\right) / \Upsilon}>0$, then for any constant $\varrho>0$ and $\|\varphi\|<\varrho$, there is $t^{\prime}=t^{\prime}(\varrho)>0$, such that $e^{-\beta t} E\left\{V\left(x\left(t_{0}\right)\right)\right\}<1$ for all $t \geq t^{\prime}$. According to Definition 3 , we have $E\left\|x\left(t, t_{0}, \varphi\right)\right\|<\widetilde{B}$ for all $t \geq t^{\prime}$. In other words, system (3) is mean-square uniformly ultimately bounded. This completes the proof.
Note from (25), we know that there exists a constant vector $\mathfrak{B}$, such that

$$
E\left\{V\left(x\left(t_{0}\right)\right)\right\} \leq \mathfrak{B} E\left\|x\left(t_{0}\right)\right\|^{2} e^{-\beta t_{0}} .
$$

Thus, combining (50) and (52) leads to

$$
\begin{aligned}
E\|x(t)\|^{2} & \leq \frac{e^{-\beta\left(t-t_{0}\right)} \mathfrak{B} E\left\|x\left(t_{0}\right)\right\|^{2}+\beta^{-1} \bar{\alpha}^{2} J^{T} P J+H}{\Upsilon} \\
& =\frac{e^{-\beta\left(t-t_{0}\right)} \mathfrak{B} E\left\|x\left(t_{0}\right)\right\|^{2}}{\Upsilon}+\frac{\beta^{-1} \bar{\alpha}^{2} J^{T} P J+H}{\Upsilon} \\
& \leq \frac{e^{-\beta\left(t-t_{0}\right)} \mathfrak{B} E\left\|x\left(t_{0}\right)\right\|^{2}}{\Upsilon}+N,
\end{aligned}
$$

where $N=\left(\beta^{-1} \bar{\alpha}^{2} J^{T} P J+H\right) / \Upsilon$.

Theorem 11. If all of the conditions of Theorem 10 hold, then there exists an attractor $\mathbb{A}_{\widetilde{B}}$ for the solutions of system (3), where $\mathbb{A}_{\widetilde{B}}=\left\{\varphi \in L_{\mathscr{F}_{0}}^{b} \mid E\|\varphi(s)\|_{\infty} \leq \widetilde{B}, t \geq t_{0}\right\}$.

Proof. If one choose $\widetilde{B}=\sqrt{\left(1+\beta^{-1} \bar{\alpha}^{2} J^{T} P J\right) / \Upsilon}>0$, Theorem 10 shows that for any $\phi$, there is $t^{\prime}>0$, such that $E\left\|x\left(t, t_{0}, \phi\right)\right\|<\widetilde{B}$ for all $t \geq t^{\prime}$. Let $\mathbb{A}_{\widetilde{B}}=\left\{\varphi \in L_{\mathscr{F}_{0}}^{b} \mid\right.$ $\left.E\|\varphi(s)\|_{\infty} \leq \widetilde{B}, t \geq t_{0}\right\}$. Clearly, $\mathbb{A}_{\widetilde{B}}$ is closed, bounded, and invariant. Furthermore, $\lim _{t \rightarrow \infty} \operatorname{supinf}_{y \in \mathbb{A}_{\tilde{B}}}\|x(t ; 0, \phi)-y\|=$ 0 . Therefore, $A_{\widetilde{B}}$ is a stochastic attractor for the solutions of system (3).

Corollary 12. In addition to all of the conditions of Theorem 10 that hold, if $J=0$ and $F_{j}(0)=0$, then system (3) has a trivial solution $x(t) \equiv 0$, and the trivial solution of system (3) is meansquare exponentially stable. 
Proof. If $J=0$ and $F_{j}(0)=0$, then it is obvious that system (3) has a trivial solution $x(t) \equiv 0$. From Theorem 10 , one has

$$
E\|x(t ; 0, \phi)\|^{2} \leq K e^{-\beta t}, \quad \forall \phi,
$$

where $K=E\|V(x(0))\|^{2} / \Upsilon$. Therefore, the trivial solution of system (3) is mean-square exponentially stable. This completes the proof.

In practice, parameter uncertainties in neural networks are always unavoidable, in order to explain such a phenomenon. In this section, we will investigate the mean-square uniform ultimate boundedness of the switching stochastic systems with uncertainties by applying the average dwell time.

Now, we consider the switched stochastic Cohen-Grossberg neural networks with unknown parameters as follows:

$$
\begin{aligned}
& d x(t)=-\widehat{\alpha}(x(t))\left[\widehat{\beta}(x(t))-\left(A_{\sigma}(t)+\Delta A_{\sigma}(t)\right)\right. \\
& \cdot F(x(t))-\left(B_{\sigma}(t)+\Delta B_{\sigma}(t)\right) F(x(t-\tau(t))) \\
& -\left(C_{\sigma}(t)+\Delta C_{\sigma}(t)\right)
\end{aligned}
$$$$
\Delta_{1 i}=\left[\begin{array}{cccccccccccc}
\phi_{i 11} & 0 & 0 & 0 & \phi_{i 15} & 0 & 0 & \phi_{i 18} & \phi_{i 19} & 0 & 0 & 0 \\
* & \phi_{i 22} & 0 & 0 & 0 & \phi_{i 26} & 0 & \phi_{i 28} & 0 & 0 & 0 & 0 \\
* & * & \phi_{i 33} & 0 & 0 & 0 & \phi_{i 37} & 0 & \phi_{i 39} & 0 & 0 & 0 \\
* & * & * & \phi_{i 44} & \phi_{i 45} & 0 & 0 & \phi_{i 48} & \phi_{i 49} & 0 & 0 & 0 \\
* & * & * & * & \bar{\phi}_{i 55} & 0 & 0 & 0 & 0 & 0 & 0 & 0 \\
* & * & * & * & * & \phi_{i 66} & 0 & 0 & 0 & 0 & 0 & 0 \\
* & * & * & * & * & * & \phi_{i 77} & 0 & 0 & 0 & 0 & 0 \\
* & * & * & * & * & * & * & \widetilde{\phi}_{i 88} & 0 & 0 & 0 & 0 \\
* & * & * & * & * & * & * & * & \widetilde{\phi}_{i 99} & 0 & 0 & 0 \\
* & * & * & * & * & * & * & * & * & \phi_{i 10} & 0 & 0 \\
* & * & * & * & * & * & * & * & * & * & \bar{\phi}_{i 11} & 0 \\
* & * & * & * & * & * & * & * & * & * & * & \bar{\phi}_{i 12}
\end{array}\right]<
$$

$$
\begin{aligned}
\phi_{i 11} & =\frac{1}{\bar{a}^{2}}\left[\beta P_{i}-2 \Omega_{1} P_{i} \Omega_{2}+\lambda \Pi_{1}^{T} \Pi_{1}+\varepsilon \Omega_{4} D_{1}+P_{i}+\frac{1}{4 \beta^{2}} I+\tau_{M} e^{\beta \tau_{M}} Q_{i 1}+\tau_{m} e^{\beta \tau_{m}} Q_{i 2}+e^{\beta \tau_{M}} Q_{i 3}+\tau_{M} R_{i}+\left(\tau_{M}-\tau_{m}\right) S_{i}\right. \\
& \left.+\frac{\tau_{M}^{2}}{4} Z_{i}+\frac{\tau_{M}^{2}}{4} \widetilde{Z}_{i}-\Omega_{3} D_{1}\right] \\
\phi_{i 15} & =P_{i} A_{i} \\
\phi_{i 18} & =P_{i} B_{i} \\
\phi_{i 19} & =P_{i} C_{i} \\
\phi_{i 22} & =-\tau_{M} Q_{i 1} \\
\phi_{i 26} & =\phi_{i 28}=0 \\
\phi_{i 33} & =-\tau_{m} Q_{i 2}
\end{aligned}
$$

Theorem 13. For a given constant $\beta>0, \varepsilon>0, \varepsilon_{1}>0$, $\varepsilon_{2}>0, \varepsilon_{3}>0$, if there exist positive scalars $\lambda_{i}$, positive-definite matrix $P_{i}=\operatorname{diag}\left(p_{i 1}, p_{i 2}, \ldots, p_{\text {in }}\right), L=\operatorname{diag}\left(l_{i 1}, l_{i 2}, \ldots, l_{\text {in }}\right)$, $D_{i}=\operatorname{diag}\left(D_{i 1}, D_{i 2}, \ldots, D_{i n}\right),(i=1,2), Q_{i 1}, Q_{i 2}, Q_{i 3}, Q_{i 4}, R_{i}$, $S_{i}, \widetilde{Z}_{i}, Z_{i}, U_{i}$, and any matrices $\bar{M}_{i 1}, \bar{M}_{i 2}, \bar{M}_{i 3}$ with appropriate dimensions such that the following condition holds:

$$
\begin{gathered}
{\left[\begin{array}{cccc}
\Delta_{1 i} & \bar{M}_{i 1} & \bar{M}_{i 2} & \bar{M}_{i 3} \\
* & -\varepsilon_{1} I & 0 & 0 \\
* & * & -\varepsilon_{2} I & 0 \\
* & * & * & -\varepsilon_{3} I
\end{array}\right]<0,} \\
\\
\quad P_{i}<\lambda_{i} I,
\end{gathered}
$$

where 


$$
\begin{aligned}
& \phi_{i 37}=\phi_{i 39}=0, \\
& \phi_{i 44}=\lambda \Pi_{2}^{T} \Pi_{2}-\Omega_{3} D_{2}+\frac{1}{4 \beta^{2}} I-(1-\mu) Q_{i 3}, \\
& \phi_{i 45}=0 \text {, } \\
& \phi_{i 48}=\Omega_{4} D_{2} \text {, } \\
& \phi_{i 49}=0 \text {, } \\
& \tilde{\phi}_{i 55}=e^{\beta \tau_{M}} Q_{i 4}+L K(\iota)+\varepsilon^{-1} \Omega_{4} D_{1}+\tau_{M}^{2} U_{i}-D_{1}+\frac{1}{\beta^{2}} I+\varepsilon_{1} E_{A_{i}}^{T} E_{A_{i}}, \\
& \phi_{i 66}=-\frac{e^{-\beta \tau_{M}}}{\tau_{M}} R \\
& \phi_{i 77}=-\frac{e^{-\beta \tau_{M}}}{\tau_{M}-\tau_{m}} S_{i}, \\
& \widetilde{\phi}_{i 88}=-(1-\mu) Q_{i 4}-D_{2}+\frac{1}{\beta^{2}} I+\varepsilon_{2} E_{B_{i}}^{T} E_{B_{i}}, \\
& \widetilde{\phi}_{i 99}=-L_{i}+\varepsilon_{3} E_{C_{i}}^{T} E_{C_{i}} \text {, } \\
& \phi_{i 10}=-(1-\mu) e^{-\beta \tau_{M}} U_{i} \text {, } \\
& \bar{\phi}_{i 11}=-e^{-\beta \tau_{M}} Z_{i} \text {, } \\
& \bar{\phi}_{i 12}=-e^{-\beta \tau_{M}} \widetilde{Z}_{i} \text {, } \\
& \bar{M}_{i 1}=\left[\begin{array}{llllllllllll}
P_{i} H_{A_{i}} & 0 & 0 & 0 & 0 & 0 & 0 & 0 & 0 & 0 & 0 & 0
\end{array}\right]^{T} \text {, } \\
& \bar{M}_{i 2}=\left[\begin{array}{lllllllllllll}
P_{i} H_{B_{i}} & 0 & 0 & 0 & 0 & 0 & 0 & 0 & 0 & 0 & 0 & 0 & 0
\end{array}\right]^{T} \text {, } \\
& \bar{M}_{i 3}=\left[\begin{array}{llllllllllllll}
P_{i} H_{C_{i}} & 0 & 0 & 0 & 0 & 0 & 0 & 0 & 0 & 0 & 0 & 0 & 0 & 0
\end{array}\right]^{T} \text {, }
\end{aligned}
$$

then system (55) is mean-square uniformly ultimately bounded for any switching signal with average dwell time satisfying

$$
\tau_{a}>\tau_{a}^{*}=\frac{\ln M_{\max }}{\beta},
$$

where $M_{\max }=\mathfrak{B}_{\text {max }} / \Upsilon_{\min }, \mathfrak{B}_{\max }=\max _{k \in \bar{N}, 1 \leq i \leq n}\left\{\mathfrak{B}_{i_{k}}\right\}, \Upsilon_{\text {min }}=$ $\min _{i_{k}}\left\{Y_{i_{k}}\right\}$.

Proof. Let us consider the same Lyapunov functional candidate

$$
\begin{aligned}
& V_{\sigma}(t)=e^{\beta t} x^{T}(t) P_{\sigma(t)} x(t) \\
& +\tau_{M} \int_{t-\tau_{M}}^{t} e^{\beta\left(s+\tau_{M}\right)} x^{T}(s) Q_{1_{\sigma(t)}} x(s) d s \\
& +\tau_{m} \int_{t-\tau_{m}}^{t} e^{\beta\left(s+\tau_{m}\right)} x^{T}(s) Q_{2_{\sigma(t)}} x(s) d s \\
& +\int_{t-\tau(t)}^{t} e^{\beta\left(s+\tau_{M}\right)} x^{T}(s) Q_{3_{\sigma(t)}} x(s) d s \\
& +\int_{t-\tau(t)}^{t} e^{\beta\left(s+\tau_{M}\right)} F^{T}(x(s)) Q_{4_{\sigma(t)}} F(x(s)) d s
\end{aligned}
$$

$$
\begin{aligned}
& +\int_{-\tau_{M}}^{0} \int_{t+\theta}^{t} e^{\beta s} x^{T}(s) R_{\sigma(t)} x(s) d s d \theta \\
& +\int_{-\tau_{M}}^{-\tau_{m}} \int_{t+\theta}^{t} e^{\beta s} x^{T}(s) S_{\sigma(t)} x(s) d s d \theta \\
& +\tau_{M} \int_{-\tau(t)}^{0} \int_{t+\theta}^{t} e^{\beta s} F^{T}(x(s)) U_{\sigma(t)} F(x(s)) d s d \theta \\
& +\frac{\tau_{M}^{2}}{2} \int_{-\tau_{M}}^{0} \int_{\theta}^{0} \int_{t+\lambda}^{t} e^{\beta s} x^{T}(s) Z_{\sigma(t)} x(s) d s d \lambda d \theta \\
& +\frac{\left(\tau_{M}^{2}-\tau_{m}^{2}\right)}{2} \\
& \cdot \int_{-\tau_{M}}^{-\tau_{m}} \int_{\theta}^{0} \int_{t+\lambda}^{t} e^{\beta s} x^{T}(s) \widetilde{Z}_{\sigma(t)} x(s) d s d \lambda d \theta \\
& +\sum_{j=1}^{n} l_{j_{\sigma(t)}} \int_{0}^{\infty} k_{j}(\delta) \int_{t-\delta}^{t} e^{\beta(\gamma+\delta)} F_{j}^{2}\left(x_{j}(\gamma)\right) d \gamma d \delta .
\end{aligned}
$$

Then, we will compute the stochastic derivative of $V(t)$ along the trajectory of system (55). Therefore, from 
Theorem 10, we merely need to obtain the idea of the following equalities:

$$
\begin{aligned}
& 2 e^{\beta t} x^{T}(t) P_{i} \alpha(x(t)) \Delta A_{i}(t) F(x(t)) \\
& \quad=2 e^{\beta t} P_{i} \alpha(x(t)) H_{A_{i}} G_{A_{i}}(t) E_{A_{i}} F(x(t)), \\
& 2 e^{\beta t} x^{T}(t) P_{i} \alpha(x(t)) \Delta B_{i}(t) F(x(t-\tau(t))) \\
& =2 e^{\beta t} P_{i} \alpha(x(t)) H_{B_{i}} G_{B_{i}}(t) E_{B_{i}} F(x(t-\tau(t))), \\
& 2 e^{\beta t} x^{T}(t) P_{i} \alpha(x(t)) \Delta C_{i}(t) \int_{-\infty}^{t} K(t-s) F(x(s)) d s \\
& =2 e^{\beta t} P_{i} \alpha(x(t)) H_{C_{i}} G_{C_{i}}(t) \\
& \cdot E_{C_{i}} \int_{-\infty}^{t} K(t-s) F(x(s)) d s .
\end{aligned}
$$

Moreover, by assumption (H5) and Lemma 7, we obtain

$$
\begin{aligned}
& 2 e^{\beta t} x^{T}(t) P_{i} \alpha(x(t)) H_{A_{i}} G_{A_{i}}(t) E_{A_{i}} F(x(t)) \\
& \quad \leq e^{\beta t} \varepsilon_{1}^{-1} \bar{\alpha}^{2} x^{T}(t) P_{i} H_{A_{i}} H_{A_{i}}^{T} P_{i} x(t)+e^{\beta t} \varepsilon_{1} F^{T}(x(t))
\end{aligned}
$$

$$
\begin{aligned}
& \cdot E_{A_{i}}^{T} E_{A_{i}} F(x(t)), \\
& 2 e^{\beta t} x^{T}(t) P_{i} \alpha(x(t)) H_{B_{i}} G_{B_{i}}(t) E_{B_{i}} F(x(t-\tau(t))) \\
& \leq e^{\beta t} \varepsilon_{2}^{-1} \bar{\alpha}^{2} x^{T}(t) P_{i} H_{B_{i}} H_{B_{i}}^{T} P_{i} x(t) \\
& +e^{\beta t} \varepsilon_{1} F^{T}\left(x(t-\tau(t)) E_{B_{i}}^{T} E_{B_{i}} F(x(t-\tau(t)))\right), \\
& 2 e^{\beta t} P_{i} \alpha(x(t)) H_{C_{i}} G_{C_{i}}(t) E_{C_{i}} \int_{-\infty}^{t} K(t-s) F(x(s)) d s \\
& \leq e^{\beta t} \varepsilon_{3}^{-1} \bar{\alpha}^{2} x^{T}(t) P_{i} H_{C_{i}} H_{C_{i}}^{T} P_{i} x(t) \\
& +e^{\beta t} \varepsilon_{3}\left(\int_{-\infty}^{t} K(t-s) F(x(s)) d s\right)^{T} \\
& \cdot E_{B_{i}}^{T} E_{B_{i}} \int_{-\infty}^{t} K(t-s) F(x(s)) d s .
\end{aligned}
$$

Then, along a similar way to Theorem 10, it can be deduced that

$$
\widetilde{\Delta}_{1 i}=\left[\begin{array}{cccccccccccc}
\tilde{\phi}_{i 11} & 0 & 0 & 0 & \tilde{\phi}_{i 15} & 0 & 0 & \tilde{\phi}_{i 18} & \tilde{\phi}_{i 19} & 0 & 0 & 0 \\
* & \phi_{i 22} & 0 & 0 & 0 & \phi_{i 26} & 0 & \phi_{i 28} & 0 & 0 & 0 & 0 \\
* & * & \phi_{i 33} & 0 & 0 & 0 & \phi_{i 37} & 0 & \phi_{i 39} & 0 & 0 & 0 \\
* & * & * & \phi_{i 44} & \phi_{i 45} & 0 & 0 & \phi_{i 48} & \phi_{i 49} & 0 & 0 & 0 \\
* & * & * & * & \tilde{\phi}_{i 55} & 0 & 0 & 0 & 0 & 0 & 0 & 0 \\
* & * & * & * & * & \phi_{i 66} & 0 & 0 & 0 & 0 & 0 & 0 \\
* & * & * & * & * & * & \phi_{i 77} & 0 & 0 & 0 & 0 & 0 \\
* & * & * & * & * & * & * & \widetilde{\phi}_{i 88} & 0 & 0 & 0 & 0 \\
* & * & * & * & * & * & * & * & \widetilde{\phi}_{i 99} & 0 & 0 & 0 \\
* & * & * & * & * & * & * & * & * & \phi_{i 10} & 0 & 0 \\
* & * & * & * & * & * & * & * & * & * & \bar{\phi}_{i 11} & 0 \\
* & * & * & * & * & * & * & * & * & * & * & \bar{\phi}_{i 12}
\end{array}\right],
$$

$P_{i}<\lambda_{i} I$,

$$
\begin{aligned}
\widetilde{\phi}_{i 11} & =\beta P_{i}-2 \Omega_{1} P_{i} \Omega_{2}+\lambda \Pi_{1}^{T} \Pi_{1}+\varepsilon \Omega_{4} D_{1}+P_{i}+\frac{1}{4 \beta^{2}} I+\tau_{M} e^{\beta \tau_{M}} Q_{i 1}+\tau_{m} e^{\beta \tau_{m}} Q_{i 2}+e^{\beta \tau_{M}} Q_{i 3}+\tau_{M} R_{i}+\left(\tau_{M}-\tau_{m}\right) S_{i} \\
& +\frac{\tau_{M}^{2}}{4} Z_{i}+\frac{\tau_{M}^{2}}{4} \widetilde{Z}_{i}-\Omega_{3} D_{1}+\varepsilon_{1}^{-1} \bar{\alpha}^{2} P_{i} H_{A_{i}} H_{A_{i}}^{T} P_{i}+\varepsilon_{2}^{-1} \bar{\alpha}^{2} P_{i} H_{B_{i}} H_{B_{i}}^{T} P_{i}+\varepsilon_{3}^{-1} \bar{\alpha}^{2} P_{i} H_{C_{i}} H_{C_{i}}^{T} P_{i}, \\
\widetilde{\phi}_{i 15} & =P_{i} \alpha(x(t)) A_{i}, \\
\widetilde{\phi}_{i 18} & =P_{i} \alpha(x(t)) B_{i}, \\
\tilde{\phi}_{i 19} & =P_{i} \alpha(x(t)) C_{i} .
\end{aligned}
$$


By using Schur's complement lemma, the LMI (56) is equivalent to

$$
\underline{\Delta}_{1 i}=\left[\begin{array}{cccccccccccc}
\phi_{i 11} & 0 & 0 & 0 & \phi_{i 15} & 0 & 0 & \phi_{i 18} & \phi_{i 19} & 0 & 0 & 0 \\
* & \phi_{i 22} & 0 & 0 & 0 & \phi_{i 26} & 0 & \phi_{i 28} & 0 & 0 & 0 & 0 \\
* & * & \phi_{i 33} & 0 & 0 & 0 & \phi_{i 37} & 0 & \phi_{i 39} & 0 & 0 & 0 \\
* & * & * & \phi_{i 44} & \phi_{i 45} & 0 & 0 & \phi_{i 48} & \phi_{i 49} & 0 & 0 & 0 \\
* & * & * & * & \tilde{\phi}_{i 55} & 0 & 0 & 0 & 0 & 0 & 0 & 0 \\
* & * & * & * & * & \phi_{i 66} & 0 & 0 & 0 & 0 & 0 & 0 \\
* & * & * & * & * & * & * & \widetilde{\phi}_{i 88} & 0 & 0 & 0 & 0 \\
* & * & * & * & * & * & * & * & \widetilde{\phi}_{i 99} & 0 & 0 & 0 \\
* & * & * & * & * & * & * & * & * & \phi_{i 10} & 0 & 0 \\
* & * & * & * & * & * & * & * & * & * & \bar{\phi}_{i 11} & 0 \\
* & * & * & * & * & * & * & * & * & * & * & \bar{\phi}_{i 12}
\end{array}\right]<0,
$$

where

$$
\begin{aligned}
\underline{\phi}_{i 11} & =\frac{1}{\bar{\alpha}^{2}}\left[\beta P_{i}-2 \Omega_{1} P_{i} \Omega_{2}+\lambda \Pi_{1}^{T} \Pi_{1}+\varepsilon \Omega_{4} D_{1}+P_{i}\right. \\
& +\frac{1}{4 \beta^{2}} I+\tau_{M} e^{\beta \tau_{M}} Q_{i 1}+\tau_{m} e^{\beta \tau_{m}} Q_{i 2}+e^{\beta \tau_{M}} Q_{i 3} \\
& \left.+\tau_{M} R_{i}+\left(\tau_{M}-\tau_{m}\right) S_{i}+\frac{\tau_{M}^{2}}{4} Z_{i}+\frac{\tau_{M}^{2}}{4} \widetilde{Z}_{i}-\Omega_{3} D_{1}\right] \\
& +\varepsilon_{1}^{-1} P_{i} H_{A_{i}} H_{A_{i}}^{T} P_{i}+\varepsilon_{2}^{-1} P_{i} H_{B_{i}} H_{B_{i}}^{T} P_{i} \\
& +\varepsilon_{3}^{-1} P_{i} H_{C_{i}} H_{C_{i}}^{T} P_{i}, \\
\phi_{i 15} & =P A, \\
\phi_{i 18} & =P B \\
\phi_{i 19} & =P C .
\end{aligned}
$$

Supposing $\alpha(x(t)) \alpha(x(t)) \leq \bar{\alpha}^{2} I$ and multiplying both sides of LMI (64) by

$$
\operatorname{diag}\{\alpha(x(t)), I, I, I, I, I, I, I, I, I, I, I\},
$$

we derive the LMI $\widetilde{\Delta}_{1 i}<0$.

Therefore, when $t \in\left[t_{k}, t_{k+1}\right)$, the $i_{k}$ th subsystem is activated. From the proof of Theorem 10 and (53), there exists a positive constant vector $\mathfrak{B}_{i_{k}}$, such that

$$
\begin{aligned}
E\|x(t)\|^{2} & \\
& \leq \frac{\mathfrak{B}_{i_{k}} E\left\|x\left(t_{k}\right)\right\|^{2} e^{-\beta\left(t-t_{k}\right)}+\beta^{-1} \bar{\alpha}^{2} J^{T} P J+H}{\Upsilon_{i_{k}}} \\
& =M_{i_{k}} E\left\|x\left(t_{k}\right)\right\|^{2} e^{-\beta\left(t-t_{k}\right)}+N_{i_{k}},
\end{aligned}
$$

where $M_{i_{k}}=\mathfrak{B}_{i_{k}} / \Upsilon_{i_{k}}, \Upsilon_{i_{k}}=\min _{k \in \bar{N}, 1 \leq i \leq n}\left\{\lambda_{\min }\left(P_{i}\right)\right\}, N_{i_{k}}=$ $\left(\beta^{-1} \bar{\alpha}^{2} J^{T} P J+H\right) / \Upsilon_{i_{k}}$.

As the system state is continuous, it follows from (67) that

$$
\begin{aligned}
& E\|x(t)\|^{2} \\
& \leq \frac{\mathfrak{B}_{i_{k}} E\left\|x\left(t_{k}\right)\right\|^{2} e^{-\beta\left(t-t_{k}\right)}+\beta^{-1} \bar{\alpha}^{2} J^{T} P J+H}{\Upsilon_{i_{k}}} \\
& =M_{i_{k}} E\left\|x\left(t_{k}\right)\right\|^{2} e^{-\beta\left(t-t_{k}\right)}+N_{i_{k}} \leq \cdots \\
& \leq e^{\sum_{v=0}^{k} \ln M_{i_{v}}-\beta\left(t-t_{0}\right)} E\left\|x\left(t_{0}\right)\right\|^{2}+\left[M_{i_{k}} e^{-\beta\left(t-t_{k}\right)} N_{i_{k}}\right. \\
& +M_{i_{k}} M_{i_{k-1}} e^{-\beta\left(t-t_{k-1}\right)} N_{i_{k-1}} \\
& +M_{i_{k}} M_{i_{k-1}} M_{i_{k-2}} e^{-\beta\left(t-t_{k-2}\right)} N_{i_{k-2}}+\cdots \\
& \left.+M_{i_{k}} M_{i_{k-1}} M_{i_{k-2}} \cdots M_{i_{1}} e^{-\beta\left(t-t_{1}\right)} N_{i_{1}}+N_{i_{k}}\right] \\
& \leq e^{(k+1) \ln M_{\max }-\beta\left(t-t_{0}\right)} E\left\|x\left(t_{0}\right)\right\|^{2}+\left[M_{\max }^{k} N_{\max }\right. \\
& +M_{\max }^{(k-1)} N_{\max }+M_{\max }^{(k-2)} N_{\max }+\cdots+M_{\max }^{2} N_{\max } \\
& \left.+M_{\max } N_{\max }+N_{\max }\right] \\
& \leq M_{\max } e^{k \ln M_{\max }-\beta\left(t-t_{0}\right)} E\left\|x\left(t_{0}\right)\right\|^{2} \\
& +\frac{N_{\max }\left(1-M_{\max }^{(k+1)}\right)}{1-M_{\max }} \\
& \leq M_{\max } e^{\ln M_{\max } N_{\sigma}\left(t-t_{0}\right)-\beta\left(t-t_{0}\right)} E\left\|x\left(t_{0}\right)\right\|^{2} \\
& +\frac{N_{\max }\left(1-M_{\max }^{(k+1)}\right)}{1-M_{\max }}
\end{aligned}
$$




$$
\begin{aligned}
& \leq M_{\max } e^{N_{0} \ln M_{\max }-\left(\beta-\ln M_{\max } / T_{\alpha}\right)\left(t-t_{0}\right)} E\left\|x\left(t_{0}\right)\right\|^{2} \\
& +\frac{N_{\max }\left(1-M_{\max }^{(k+1)}\right)}{1-M_{\max }} \\
& \leq \frac{\mathfrak{B}_{\max } e^{N_{0} \ln M_{\max }-\left(\beta-\ln M_{\max } / T_{\alpha}\right)\left(t-t_{0}\right)} E\left\|x\left(t_{0}\right)\right\|^{2}}{\Upsilon_{\min }} \\
& +\frac{\left(\beta^{-1} \bar{\alpha}^{2} J^{T} P J+H\right)\left(1-\mathfrak{B}_{\max }^{(k+1)} / \Upsilon_{\min }^{(k+1)}\right)}{\Upsilon_{\min }-\mathfrak{B}_{\max }} .
\end{aligned}
$$

If one chooses

$$
\begin{aligned}
\widetilde{B} & =\sqrt{\frac{1}{\Upsilon_{\min }}+\frac{\left(\beta^{-1} \bar{\alpha}^{2} J^{T} P J+H\right)\left(1-\mathfrak{B}_{\max }^{(k+1)} / \Upsilon_{\min }^{(k+1)}\right)}{\Upsilon_{\min }-\mathfrak{B}_{\max }}} \\
& >0,
\end{aligned}
$$

then for any constant $\varrho>0$ and $\|\varphi\|<\varrho$ there is $t^{\prime}=t^{\prime}(\varrho)>0$, such that $\mathfrak{B}_{\max } e^{N_{0} \ln M_{\max }-\left(\beta-\ln M_{\max } / T_{\alpha}\right)\left(t-t_{0}\right)} E\left\|x\left(t_{0}\right)\right\|^{2}<1$ for all $t \geq t^{\prime}$. According to Definition 3, we have $E\left\|x\left(t, t_{0}, \varphi\right)\right\|<$ $\widetilde{B}$ for all $t \geq t^{\prime}$. In other words, the switched stochastic Cohen-Grossberg neural networks system (55) is meansquare uniformly ultimately bounded. This completes the proof.

Theorem 14. If all of the conditions of Theorem 10 hold, then there exists an attractor $\mathbb{A}_{\widetilde{B}}$ for the solutions of system (55), where $\widetilde{\mathbb{A}}_{\widetilde{B}}=\left\{\varphi \in L_{\mathscr{F}_{0}}^{b} \mid E\|\varphi(s)\|_{\infty} \leq \widetilde{B}, t \geq t_{0}\right\}$.

Proof. If one chooses

$$
\begin{aligned}
\widetilde{B} & =\sqrt{\frac{1}{\Upsilon_{\min }}+\frac{\left(\beta^{-1} J^{T} P J+H\right)\left(1-\mathfrak{B}_{\max }^{(k+1)} / \Upsilon_{\min }^{(k+1)}\right)}{\Upsilon_{\min }-\mathfrak{B}_{\max }}} \\
& >0
\end{aligned}
$$

then Theorem 10 shows that, for any $\phi$, there is $t^{\prime}>0$; we have $E\left\|x\left(t, t_{0}, \phi\right)\right\|<\widetilde{B}$ for all $t \geq t^{\prime}$. Let $\widetilde{\mathbb{A}}_{\widetilde{B}}=\left\{\varphi \in L_{\mathscr{F}_{0}}^{b} \mid\right.$ $\left.E\|\varphi(s)\|_{\infty} \leq \widetilde{B}, t \geq t_{0}\right\}$. Clearly, $\widetilde{A}_{\widetilde{B}}$ is closed, bounded, and invariant. Furthermore, $\lim _{t \rightarrow \infty} \operatorname{sup~inf}_{y \in \widetilde{\mathbb{A}}_{\widetilde{B}}} \| x\left(t ; t_{0}, \phi\right)-$ $y \|=0$. Therefore, $\widetilde{\mathbb{A}}_{\widetilde{B}}$ is an attractor for the solutions of system (55).

Corollary 15. In addition to all of the conditions of Theorem 13 that hold, if $J=0, F_{j}(0)=0$, then system (55) has a trivial solution $x(t) \equiv 0$, and the trivial solution of system (55) is mean-square exponentially stable.

Proof. If $J=0, F_{j}(0)=0$, then it is obvious that system (55) has a trivial solution $x(t) \equiv 0$. From Theorem 10 , one has

$$
E\left\|x\left(t ; t_{0}, \phi\right)\right\|^{2} \leq \widetilde{\Upsilon} e^{-\beta\left(t-t_{0}\right)}, \quad \forall \phi,
$$

where $\tilde{\Upsilon}=\left(\mathfrak{B}_{\max } e^{N_{0} \ln M_{\max }+\left(\ln M_{\max } / T_{\alpha}\right)\left(t-t_{0}\right)} / \Upsilon_{\min }\right) E\left\|x\left(t_{0}\right)\right\|^{2}$.

Therefore, the trivial solution of system (55) is meansquare exponentially stable. This completes the proof.
Remark 16. It is noteworthy that the time-varying delay $\tau(t)$ restricts the interval $\left[\tau_{m}, \tau_{M}\right]$ and the lower bound of time delay $\tau_{m}$ may not be equal to 0 . In previous work, such as $[19,24,26,37]$, the well-used Lyapunov functional, in which the time delay information is from 0 to an upper bound $\tau_{M}$, is of the form $\int_{-\tau_{M}}^{0} \int_{t+\theta}^{t}$. In this paper, a new Lyapunov functional is constructed, which contains the information of the lower bound of time delay $\tau_{m}$, and is of the form $\int_{-\tau_{M}}^{-\tau_{m}} \int_{t+\theta}^{t}$. Thus the methods in the paper can be adopted to discuss the dynamic behaviors of interval stochastic switched CohenGrosberg neural networks with time delays. Therefore, the time-varying from $\tau_{m}$ to $\tau_{M}$ is more general and less conserving of the neural networks models. If the lower bound of time delay $\tau_{m}=0$, then our results will turn into the traditional time delay results.

Remark 17. It is known that noise disturbance is a major source of instability and poor performances in neural networks in real neural networks. If $\sigma(t, x(t), x(t-\tau(t)))=0$, the switched stochastic Cohen-Grossberg neural networks (8) degenerate into the ordinary switched Cohen-Grossberg neural networks, which have been studied for exponential stability in [22] and robust stability in [37]. In addition, when $\alpha_{i}\left(x_{i}(t)\right)=1, i=1,2, \ldots, n$, the switched Cohen-Grosberg neural networks will turn into the famous switched Hopfield neural networks; this has been investigated in [28] without distributed time delay and for global robust asymptotic stability in [32] with finite distributed time delay. However, the infinite distributed time delay was not taken into account in neural networks. Therefore, our developed results in this paper are more comfortable and general than those reported in $[28,32,37]$.

Remark 18. If $N=1$, then the switched stochastic CohenGrossberg neural networks (8) degenerate into the ordinary stochastic Cohen-Grossberg neural networks without being switched. The attractor and boundedness for stochastic Cohen-Grossberg neural networks with delays have been discussed in [35] by LaSalle-type theorem and stability has been studied in $[2,18,19]$. So our results generalize these previous results.

Remark 19. The triple integral terms

$$
\begin{aligned}
& \int_{-\tau_{M}}^{0} \int_{\theta}^{0} \int_{t+\lambda}^{t} e^{\beta s} x^{T}(s) Z x(s) d s d \lambda d \theta \\
& \int_{-\tau_{M}}^{-\tau_{m}} \int_{\theta}^{0} \int_{t+\lambda}^{t} e^{\beta s} x^{T}(s) \widetilde{Z} x(s) d s d \lambda d \theta
\end{aligned}
$$

considered in this paper lead to new dynamic criteria. We make full use of Lemma 6 and do not ignore any terms, which can reduce some conservatism of proposed method. This can be verified from the numerical examples discussed in Section 5.

Remark 20. It should be mentioned that the nonlinear output function in $[2,18,24,35,37,38]$ is required to satisfy $F_{j}(0)=$ 0 ; however, in our paper, the assumption condition was 
deleted. In assumption ( $\mathrm{H} 1)$, the constants $\delta_{i}^{-}$and $\delta_{i}^{+}$are allowed to be positive, negative, or zero, whereas the constant $\delta_{i}^{-}$is restricted to be zero or positive in $[2,18,26,35]$. Moreover, assumption (H2) is weaker than those given in $[26,35]$ since they are required to be differentiable of the amplification function $\widehat{\alpha}(x(t))$. The usual condition (H3) required to the behaved function $\widehat{\beta}(x(t))$ is differentiable in $[18,37]$ or satisfies $\left(\beta_{j}(u)-\beta_{j}(v)\right) /(u-v) \geq \gamma_{i},(u, v \in R)$ or $\beta_{i}(0)=0$ in [26]. If we take $\widehat{\beta}_{i}(x(t))=x(t) e^{|x(t) / 2|}$, obviously, the assumption (H3) in this paper holds; yet, the conditions in [26] can not be achieved.

\section{Illustrative Examples}

In this section, we present examples to show the effectiveness of the proposed method. Let $N=2$ and consider the switched stochastic neural networks with two subsystems.

Example 1. Consider the following switched stochastic Cohen-Grossberg neural network (73) with unknown parameters:

$$
\begin{aligned}
& d x(t)=-\widehat{\alpha}(x(t))\left[\widehat{\beta}(x(t))-\left(A_{i}+\Delta A_{i}(t)\right) F(x(t))\right. \\
& -\left(B_{i}+\Delta B_{i}(t)\right) F(x(t-\tau(t)))-\left(C_{i}+\Delta C_{i}(t)\right) \\
& \left.\cdot \int_{-\infty}^{t} K(t-s) F(x(s)) d s-J\right] d t \\
& +\sigma(t, x(t), x(t-\tau(t))) d \omega(t),
\end{aligned}
$$

where $\Delta A_{i}(t), \Delta B_{i}(t), \Delta C_{i}(t)$ are uncertainties, satisfying $\Delta A_{i}(t)=H_{A_{i}} G_{A_{i}}(t) E_{A_{i}}, \Delta B_{i}(t)=H_{B_{i}} G_{B_{i}}(t) E_{B_{i}}, \Delta C_{i}(t)=$ $H_{C_{i}} G_{C_{i}}(t) E_{C_{i}}$, where $G_{A_{i}}, G_{B_{i}}$, $G_{C_{i}}$ are normal bounded matrices. Let $\widehat{\alpha}(x(t))=\operatorname{diag}\left(3+\sin \left(x_{1}\right), 3+\cos \left(x_{2}\right)\right)$, $\widehat{\beta}_{i}\left(x_{i}(t)\right)=2 x_{i}(t), f_{i}\left(x_{i}(t)\right)=\tanh \left(x_{i}(t)\right)(i=1,2), K(t)=$ $\operatorname{diag}\left(2 e^{-(3 / 2) t},(1 / 2) e^{-2 t}\right), J=(2,3)^{T}$ and the connection weight matrices are as follows.

Subsystems 1. Consider

$$
\begin{aligned}
A_{1} & =\left(\begin{array}{cc}
5 & -2 \\
-1 & 3
\end{array}\right), \\
B_{1} & =\left(\begin{array}{cc}
6 & -2 \\
3 & 4
\end{array}\right), \\
C_{1} & =\left(\begin{array}{cc}
8 & 2 \\
-5 & 2
\end{array}\right), \\
E_{A_{1}} & =\left(\begin{array}{cc}
0.5 & 0 \\
0 & 0.4
\end{array}\right), \\
E_{B_{1}} & =\left(\begin{array}{cc}
0.1 & 0.3 \\
0 & 0.4
\end{array}\right), \\
E_{C_{1}} & =\left(\begin{array}{cc}
0.1 & 0 \\
0 & 0.7
\end{array}\right) .
\end{aligned}
$$

Subsystems 2. Consider

$$
\begin{aligned}
A_{2} & =\left(\begin{array}{cc}
2 & 3 \\
-2 & 7
\end{array}\right), \\
B_{2} & =\left(\begin{array}{cc}
9 & 3 \\
-3 & 4
\end{array}\right), \\
C_{2} & =\left(\begin{array}{cc}
2 & 2 \\
-1 & 5
\end{array}\right), \\
E_{A_{2}} & =\left(\begin{array}{cc}
0.1 & 0 \\
0 & 0.2
\end{array}\right), \\
E_{B_{2}} & =\left(\begin{array}{cc}
0.1 & 0 \\
0.3 & 0.2
\end{array}\right), \\
E_{C_{2}} & =\left(\begin{array}{cc}
0.3 & 0 \\
0 & 0.5
\end{array}\right), \\
\Pi_{1} & =\left(\begin{array}{cc}
0.5 & 0 \\
0 & 0.5
\end{array}\right), \\
\Pi_{2} & =\left(\begin{array}{cc}
0.1 & 0 \\
0 & 0.1
\end{array}\right), \\
H_{A_{i}} & =H_{B_{i}}=H_{C_{i}}=\left(\begin{array}{ll}
3 & 0 \\
0 & 5
\end{array}\right), \\
G_{A_{i}}(t) & =G_{B_{i}}(t)=G_{C_{i}}(t)=\left(\begin{array}{cc}
\sin t & 0 \\
0 & \cos t
\end{array}\right) .
\end{aligned}
$$

Just from assumptions (H1) and (H2), we can obtain $\alpha=$ $2, \bar{\alpha}=4, b_{i}=1.5, \delta_{i}^{-}=0, \delta_{i}^{+}=1, \tau_{m}=0.1, \tau_{M}=0.6, i=1,2$, $\mu=0.5, \iota=1, \underline{K}(1)=\operatorname{diag}(4,1 / 2)$.

Therefore, for $\beta=2$, by solving LMIS (56) and (57), we get

$$
\begin{aligned}
P_{1} & =1 \times 10^{-3}\left(\begin{array}{cc}
0.1551 & 0 \\
0 & 0.1551
\end{array}\right), \\
Q_{11} & =\left(\begin{array}{cc}
1.3273 & -0.0001 \\
-0.0001 & 1.3550
\end{array}\right) \\
Q_{21} & =\left(\begin{array}{cc}
17.8857 & -0.0011 \\
-0.0011 & 18.0732
\end{array}\right) \\
Q_{31} & =\left(\begin{array}{cc}
1.4294 & 0.0001 \\
0.0001 & 1.3633
\end{array}\right) \\
Q_{41} & =\left(\begin{array}{cc}
33.2092 & 0.0020 \\
0.0020 & 39.9381
\end{array}\right) \\
S_{1} & =\left(\begin{array}{cc}
3.7674 & -0.0002 \\
-0.0002 & 3.7960
\end{array}\right)
\end{aligned}
$$




$$
\begin{aligned}
& R_{1}=\left(\begin{array}{cc}
3.6154 & -0.0002 \\
-0.0002 & 3.6527
\end{array}\right) \text {, } \\
& U_{1}=\left(\begin{array}{rr}
42.8137 & -0.0140 \\
-0.0140 & 43.1853
\end{array}\right) \text {, } \\
& Z_{1}=\left(\begin{array}{rr}
11.3563 & -0.0003 \\
-0.0003 & 11.3904
\end{array}\right) \text {, } \\
& \widetilde{Z}_{1}=\left(\begin{array}{rr}
11.4414 & -0.0003 \\
-0.0003 & 11.4748
\end{array}\right), \\
& L_{1}=\left(\begin{array}{cc}
6.3948 & 0 \\
0 & 6.3948
\end{array}\right) \text {, } \\
& P_{2}=1 \times 10^{-3}\left(\begin{array}{cc}
0.1885 & 0 \\
0 & 0.1885
\end{array}\right) \text {, } \\
& Q_{12}=\left(\begin{array}{cc}
1.5297 & -0.0001 \\
-0.0001 & 1.6248
\end{array}\right) \text {, } \\
& Q_{22}=\left(\begin{array}{rr}
20.2951 & -0.0004 \\
-0.0004 & 20.5209
\end{array}\right) \text {, } \\
& Q_{32}=\left(\begin{array}{ll}
1.7322 & 0.0002 \\
0.0002 & 1.6554
\end{array}\right) \text {, } \\
& Q_{42}=\left(\begin{array}{cc}
40.2433 & 0.0049 \\
0.0049 & 47.6254
\end{array}\right) \text {, } \\
& S_{2}=\left(\begin{array}{cc}
4.2168 & -0.0001 \\
-0.0001 & 4.2518
\end{array}\right) \text {, } \\
& R_{2}=\left(\begin{array}{cc}
4.0988 & -0.0001 \\
-0.0001 & 4.1437
\end{array}\right) \text {, } \\
& U_{2}=\left(\begin{array}{rr}
47.0352 & -0.0358 \\
-0.0358 & 47.1122
\end{array}\right), \\
& Z_{2}=\left(\begin{array}{cc}
12.4873 & 0 \\
0 & 12.5300
\end{array}\right) \text {, } \\
& \widetilde{Z}_{2}=\left(\begin{array}{cc}
12.5778 & 0 \\
0 & 12.6198
\end{array}\right) \text {, } \\
& L_{2}=\left(\begin{array}{cc}
6.9869 & 0 \\
0 & 6.9869
\end{array}\right) \text {, } \\
& \lambda_{1}=1.78 \text {, } \\
& \lambda_{2}=2.18 \text {. }
\end{aligned}
$$

Taking $F_{j}(0)=0, J=0$ and using (59), we can obtain the average dwell time $\tau_{a}>\tau_{a}^{*}=\max \{6.6718,6.8237\}$. Therefore, one can choose $\tau_{a}=7$. The simulations of arbitrary switching signal with the average dwell time $\tau_{a}=7$ can be shown in Figure 1.

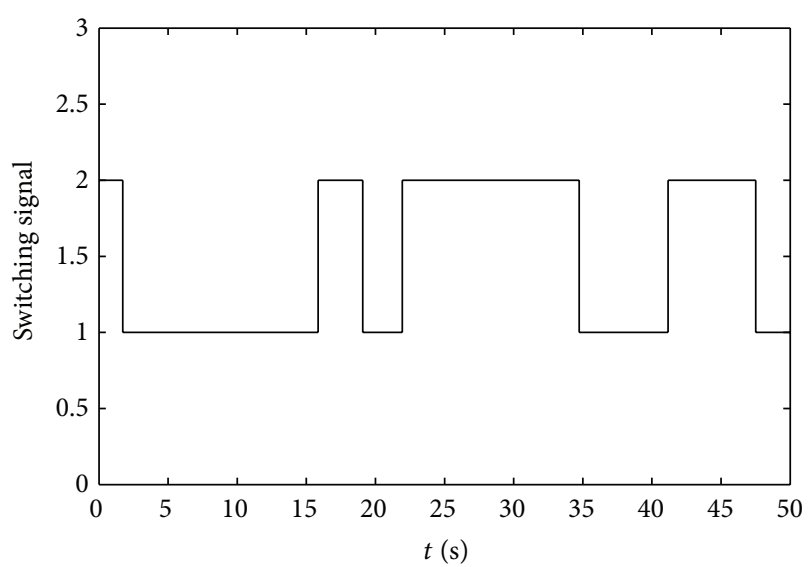

FIGURE 1: Arbitrary switching signal with the average dwell time $\tau_{a}=$ 7.

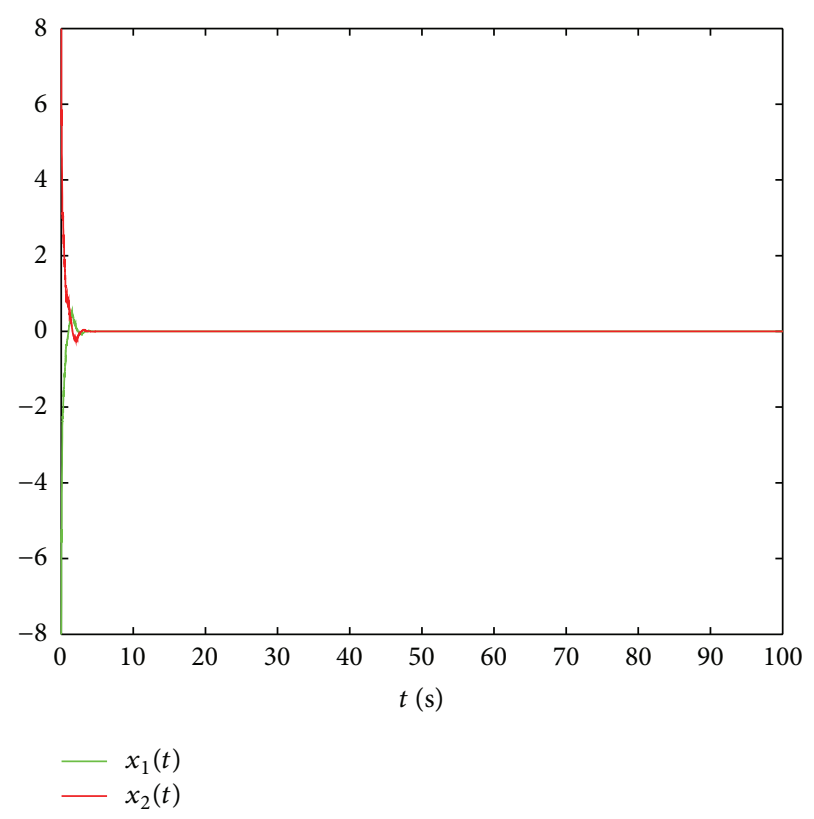

Figure 2: The mean-square exponential stability with the initial value as $x_{0}=[-8,8]$.

The mean-square exponential stability of system (55) with the initial value as $x_{0}=[-8,8]$ can be shown in Figure 2 . With the help of MATLAB, the time evolutions of state variables of the system (55) can be shown in Figure 3. In the above conditions, phase portraits of simulations under initial condition of the system (55) are shown in Figure 4.

\section{Conclusion}

This paper has studied the problem of boundedness for a class of switched stochastic Cohen-Grossberg neural networks with both average dwell time and norm-bounded parameter uncertainties. By employing multiple Lyapunov-Krasovskii functionals (25) and (60), we formulate a method that derives 


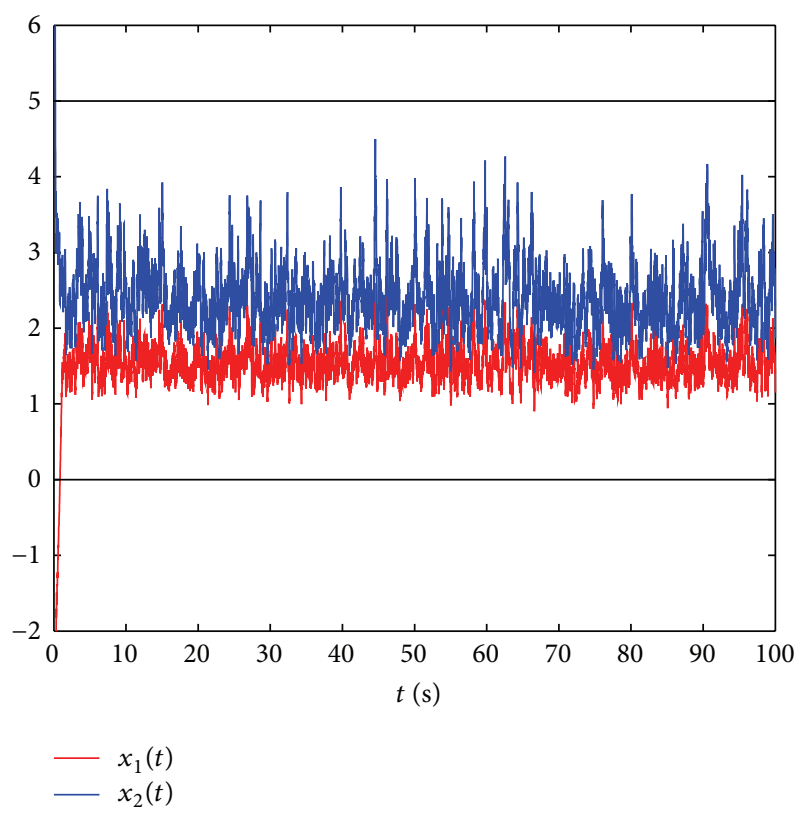

Figure 3: The mean-square uniformly ultimate boundedness.

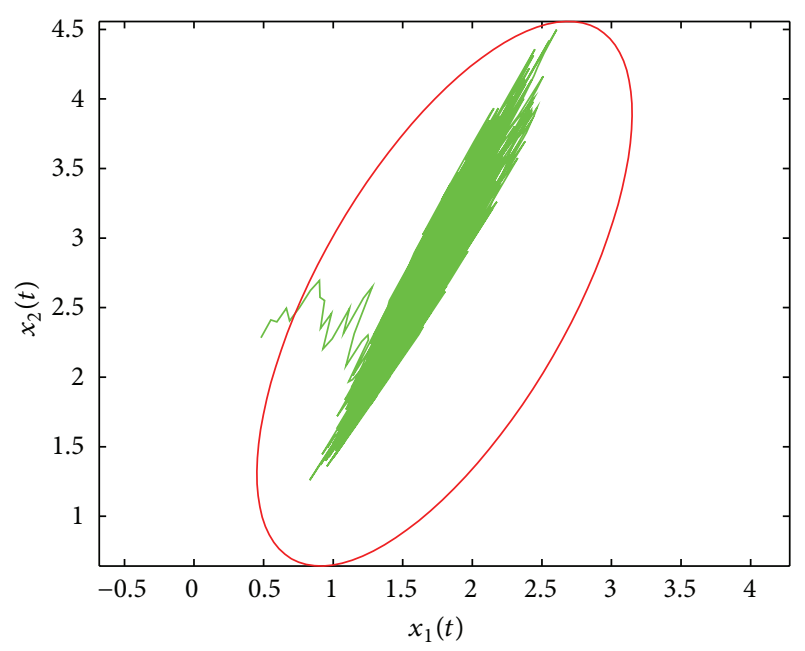

FIgURE 4: The attractor set $\mathbb{A}$.

new sufficient conditions guaranteeing the mean-square uniformly ultimate boundedness, the existence of an attractor, and the mean-square exponential stability. A numerical example has been presented to demonstrate the effectiveness and the merits of the proposed method. It is expected that the approach presented in this paper can be easily extended to analyze other neural networks.

\section{Competing Interests}

The authors declare that they have no competing interests.

\section{Acknowledgments}

The work is partially supported by the National Natural Science Foundation of China (numbers 11101053 and
71471020), China Postdoctoral Science Foundation (numbers 2014M550097 and 2015T80144), Hunan Provincial Natural Science Foundation (number 16JJ1015), Scientific Research Fund of Hunan Provincial Education Department (number 15A003), and Hunan Provincial Innovation Foundation for Postgraduate (number CX2015B373).

\section{References}

[1] C. Huang, Y. He, and L. Huang, "New results on network of neurons with delayed feedback: periodical switching of excitation and inhibition," Physics Letters A, vol. 366, no. 3, pp. 190-194, 2007.

[2] C. Huang and J. Cao, "Convergence dynamics of stochastic Cohen-Grossberg neural networks with unbounded distributed delays," IEEE Transactions on Neural Networks, vol. 22, no. 4, pp. 561-572, 2011.

[3] L. Wan and Q. Zhou, "Asymptotic behaviors of stochastic Cohen-Grossberg neural networks with mixed time-delays," Applied Mathematics and Computation, vol. 225, pp. 541-549, 2013.

[4] Q. Zhu and J. Cao, "Robust exponential stability of markovian jump impulsive stochastic Cohen-Grossberg neural networks with mixed time delays," IEEE Transactions on Neural Networks, vol. 21, no. 8, pp. 1314-1325, 2010.

[5] J. Cao and Q. Song, "Stability in Cohen-Grossberg-type bidirectional associative memory neural networks with time-varying delays," Nonlinearity, vol. 19, no. 7, pp. 1601-1617, 2006.

[6] W. Xiong and J. Cao, "Global exponential stability of discretetime Cohen-Grossberg neural networks," Neurocomputing, vol. 64, no. 1-4, pp. 433-446, 2005.

[7] Q. Zhu and J. Cao, “pth moment exponential synchronization for stochastic delayed Cohen-Grossberg neural networks with Markovian switching," Nonlinear Dynamics, vol. 67, no. 1, pp. 829-845, 2012.

[8] X. Yang, J. Cao, and W. Yu, "Exponential synchronization of memristive Cohen-Grossberg neural networks with mixed delays," Cognitive Neurodynamics, vol. 8, no. 3, pp. 239-249, 2014.

[9] R. Brause, "Medical analysis and diagnosis by neural networks," in Medical Data Analysis, pp. 1-13, Springer, Berlin, Germany, 2001.

[10] Q. Zhu, X. Li, and X. Yang, "Exponential stability for stochastic reaction-diffusion BAM neural networks with time-varying and distributed delays," Applied Mathematics and Computation, vol. 217, no. 13, pp. 6078-6091, 2011.

[11] L. Liu and Q. Zhu, "Almost sure exponential stability of numerical solutions to stochastic delay Hopfield neural networks," Applied Mathematics and Computation, vol. 266, pp. 698-712, 2015.

[12] Q. Zhu, R. Rakkiyappan, and A. Chandrasekar, "Stochastic stability of Markovian jump BAM neural networks with leakage delays and impulse control," Neurocomputing, vol. 136, pp. 136151, 2014.

[13] X. Yang and J. Cao, "Stochastic synchronization of coupled neural networks with intermittent control," Physics Letters, Section A: General, Atomic and Solid State Physics, vol. 373, no. 36, pp. 3259-3272, 2009.

[14] X. Yang, Q. Zhu, and C. Huang, "Lag stochastic synchronization of chaotic mixed time-delayed neural networks with uncertain parameters or perturbations," Neurocomputing, vol. 74, no. 10, pp. 1617-1625, 2011. 
[15] X. Yang and J. Cao, "Synchronization of discontinuous neural networks with delays via adaptive control," Discrete Dynamics in Nature and Society, vol. 2013, Article ID 147164, 9 pages, 2013.

[16] C. Huang, H. Kuang, X. Chen, and F. Wen, "An LMI approach for dynamics of switched cellular neural networks with mixed delays," Abstract and Applied Analysis, vol. 2013, Article ID 870486, 8 pages, 2013.

[17] M. A. Cohen and S. Grossberg, "Absolute stability of global pattern formation and parallel memory storage by competitive neural networks," IEEE Transactions on Systems, Man, and Cybernetics, vol. 13, no. 5, pp. 815-826, 1983.

[18] J. Cao and J. Liang, "Boundedness and stability for CohenGrossberg neural network with time-varying delays," Journal of Mathematical Analysis and Applications, vol. 296, no. 2, pp. 665685, 2004.

[19] C. Huang and J. Cao, "On pth moment exponential stability of stochastic Cohen-Grossberg neural networks with timevarying delays," Neurocomputing, vol. 73, no. 4-6, pp. 986-990, 2010.

[20] W. Xie and Q. Zhu, "Mean square exponential stability of stochastic fuzzy delayed Cohen-Grossberg neural networks with expectations in the coefficients," Neurocomputing, vol. 166, pp. 133-139, 2015.

[21] Q. Zhu and X. Li, "Exponential and almost sure exponential stability of stochastic fuzzy delayed Cohen-Grossberg neural networks," Fuzzy Sets and Systems, vol. 203, pp. 74-94, 2012.

[22] X. Yang, "Existence and global exponential stability of periodic solution for Cohen-Grossberg shunting inhibitory cellular neural networks with delays and impulses," Neurocomputing, vol. 72, no. 10-12, pp. 2219-2226, 2009.

[23] C. Huang, Z. Yang, T. Yi, and X. Zou, "On the basins of attraction for a class of delay differential equations with non-monotone bistable nonlinearities," Journal of Differential Equations, vol. 256, no. 7, pp. 2101-2114, 2014.

[24] J. Cao, G. Feng, and Y. Wang, "Multistability and multiperiodicity of delayed Cohen-Grossberg neural networks with a general class of activation functions," Physica D: Nonlinear Phenomena, vol. 237, no. 13, pp. 1734-1749, 2008.

[25] C. Huang and J. Cao, "Almost sure exponential stability of stochastic cellular neural networks with unbounded distributed delays," Neurocomputing, vol. 72, no. 13-15, pp. 3352-3356, 2009.

[26] X. Fu and X. Li, "LMI conditions for stability of impulsive stochastic Cohen-Grossberg neural networks with mixed delays," Communications in Nonlinear Science and Numerical Simulation, vol. 16, no. 1, pp. 435-454, 2011.

[27] X. Yang, C. Huang, and J. Cao, "An LMI approach for exponential synchronization of switched stochastic competitive neural networks with mixed delays," Neural Computing and Applications, vol. 21, no. 8, pp. 2033-2047, 2012.

[28] H. Huang, Y. Qu, and H.-X. Li, "Robust stability analysis of switched Hopfield neural networks with time-varying delay under uncertainty," Physics Letters A, vol. 345, no. 4-6, pp. 345354, 2005.

[29] A. Trofino, D. Assmann, C. C. Scharlau, and D. F. Coutinho, "Switching rule design for switched dynamic systems with affine vector fields," IEEE Transactions on Automatic Control, vol. 54, no. 9, pp. 2215-2222, 2009.

[30] J. Daafouz, P. Riedinger, and C. Iung, "Stability analysis and control synthesis for switched systems: a switched Lyapunov function approach," IEEE Transactions on Automatic Control, vol. 47, no. 11, pp. 1883-1887, 2002.
[31] L. Wu, Z. Feng, and W. X. Zheng, "Exponential stability analysis for delayed neural networks with switching parameters: average dwell time approach," IEEE Transactions on Neural Networks, vol. 21, no. 9, pp. 1396-1407, 2010.

[32] P. Balasubramaniam, V. Vembarasan, and R. Rakkiyappan, "Global robust asymptotic stability analysis of uncertain switched Hopfield neural networks with time delay in the leakage term," Neural Computing and Applications, vol. 21, no. 7, pp. 1593-1616, 2012.

[33] L. Xie, M. Fu, and C. E. de Souza, " $H_{\infty}$-control and quadratic stabilization of systems with parameter uncertainty via output feedback," IEEE Transactions on Automatic Control, vol. 37, no. 8, pp. 1253-1256, 1992.

[34] H. Huang and J. Cao, "Exponential stability analysis of uncertain stochastic neural networks with multiple delays," Nonlinear Analysis: Real World Applications, vol. 8, no. 2, pp. 646-653, 2007.

[35] L. Wan and Q. Zhou, "Attractor and boundedness for stochastic Cohen-Grossberg neural networks with delays," Neurocomputing, vol. 79, pp. 164-167, 2012.

[36] X. Mao and C. Yuan, Stochastic Differential Equations and Applications, Horwood, Chichester, UK, 2nd edition, 2007.

[37] K. Yuan, J. Cao, and H.-X. Li, "Robust stability of switched Cohen-Grossberg neural networks with mixed time-varying delays," IEEE Transactions on Systems, Man, and Cybernetics, Part B: Cybernetics, vol. 36, no. 6, pp. 1356-1363, 2006.

[38] B. Li and D. Xu, "Mean square asymptotic behavior of stochastic neural networks with infinitely distributed delays," Neurocomputing, vol. 72, no. 13-15, pp. 3311-3317, 2009.

[39] J. Hespanha and A. Morse, "Stability of switched systems with average dwell-time," in Proceedings of the 38th IEEE Conference on Decision and Control, vol. 3, pp. 2655-2660, Phoenix, Ariz, USA, December 1999.

[40] J. Sun, G. P. Liu, and J. Chen, "Delay-dependent stability and stabilization of neutral time-delay systems," International Journal of Robust and Nonlinear Control, vol. 19, no. 12, pp. 1364-1375, 2009.

[41] Y. Y. Wang, L. Xie, and C. E. de Souza, "Robust control of a class of uncertain nonlinear systems," Systems \& Control Letters, vol. 19, no. 2, pp. 139-149, 1992.

[42] S. Boyd, L. El Ghaoui, E. Feron, and V. Balakrishnan, Linear Matrix Inequalities in System and Control Theory, vol. 15 of Studies in Applied Mathematics, Society for Industrial and Applied Mathematics (SIAM), Philadelphia, Pa, USA, 1994. 


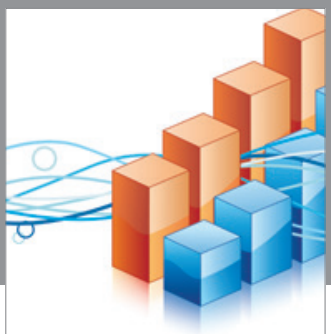

Advances in

Operations Research

vatem alat4

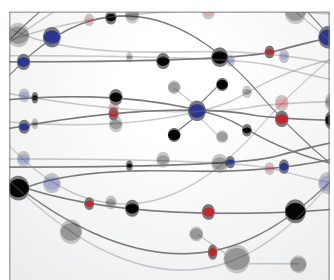

\section{The Scientific} World Journal
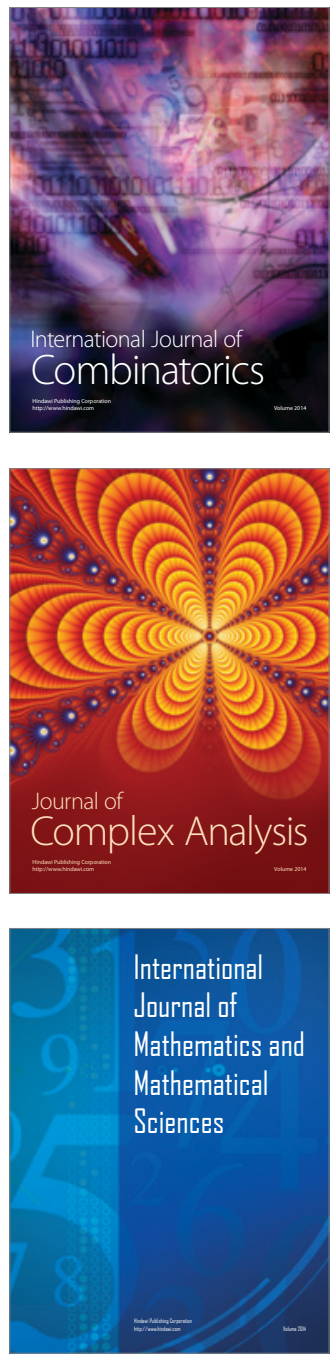
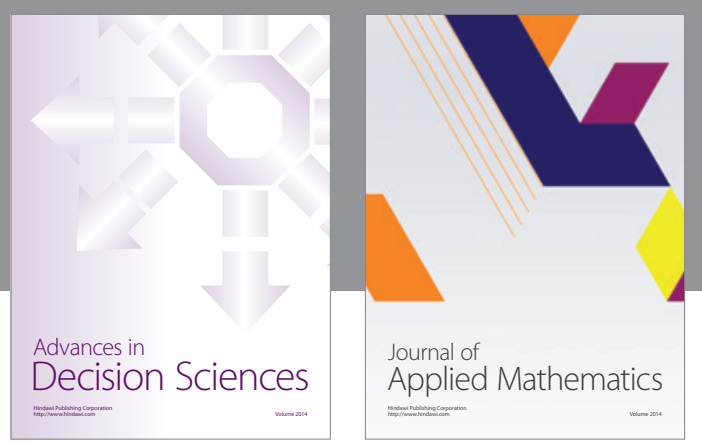

Algebra

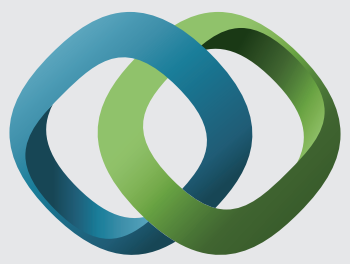

\section{Hindawi}

Submit your manuscripts at

http://www.hindawi.com
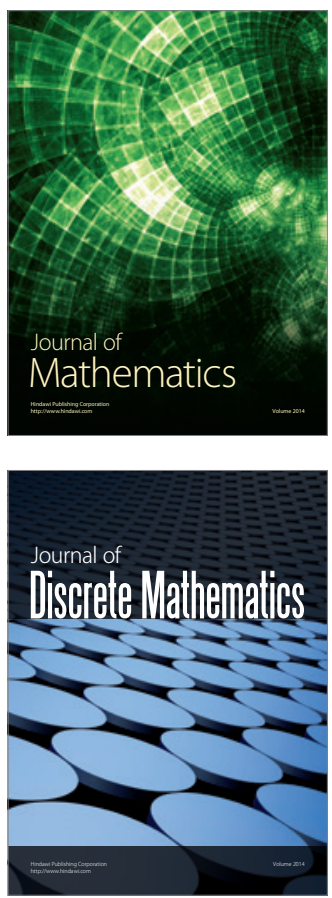

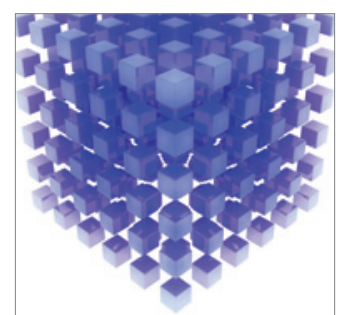

Mathematical Problems in Engineering
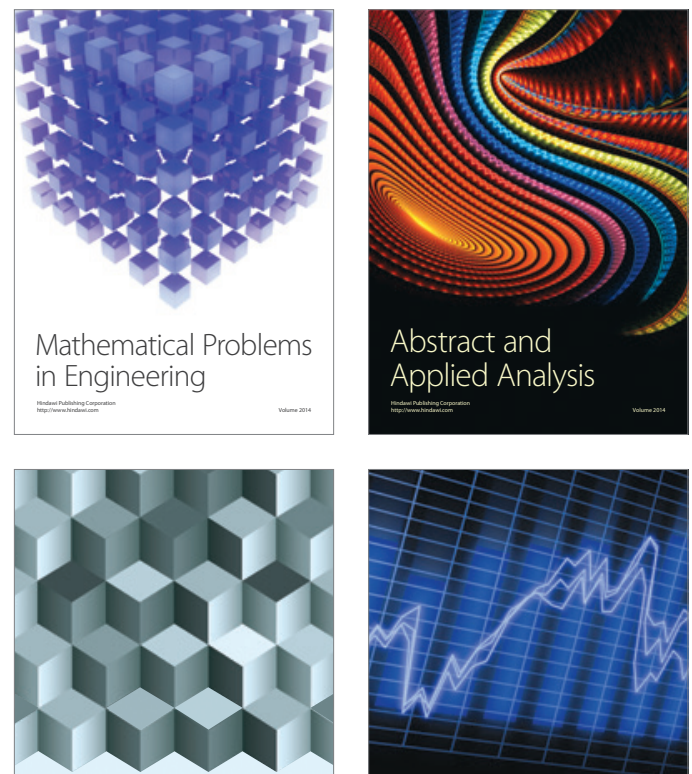

Journal of

Function Spaces

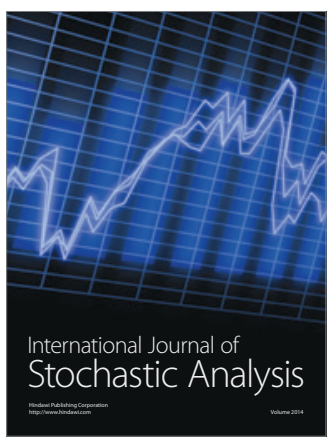

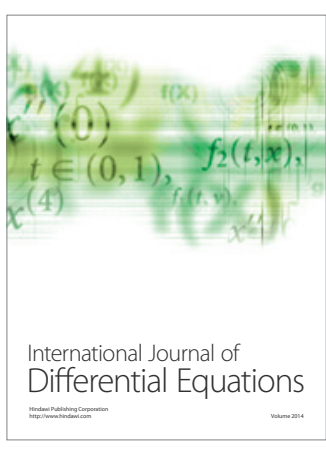
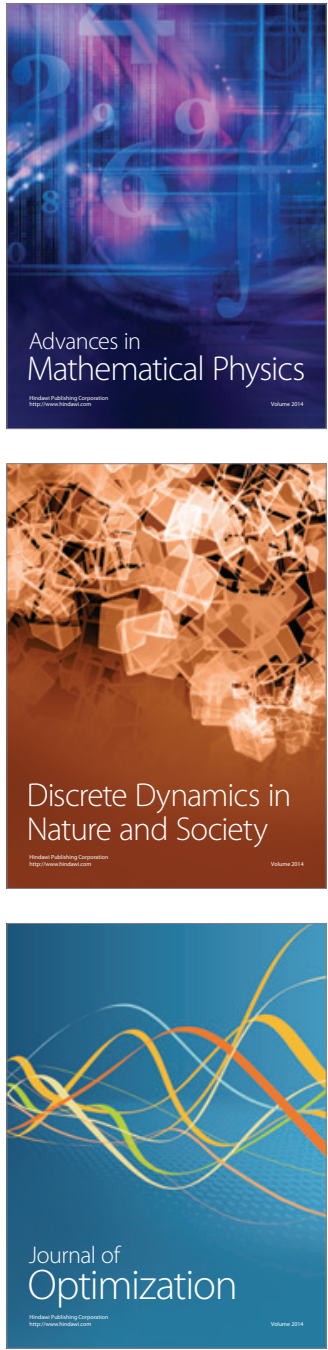